\title{
THE FOURTEEN VICTORIA DELFINO PROBLEMS AND THEIR STATUS IN THE YEAR 2020
}

\author{
ANDRÉS EDUARDO CAICEDO, BENEDIKT LÖWE
}

§1. Introduction. The Victoria Delfino problems played an important role in the development of descriptive set theory in the context of the Cabal. The first set of problems (\#1 to \# 5) were announced during one of the Very Informal Gatherings of Logicians (VIG) at UCLA in 1978. They were subsequently published as an Appendix [KM78A] in [CABAL i] with the following explanations and rules:

The following list of problems was distributed during a very informal gathering of logicians at UCLA in January 1978. We are reproducing it here because of its obvious relevance to the contents of this volume.

A cash prize of $\$ 100$ is offered by the logicians in the Los Angeles area for the solution of each of the following five problems. This competition is financed by the Victoria Delfino Fund for the Advancement of Logic which was established by a generous contribution from Miss Victoria Delfino.

Employees of UCLA and Caltech and their immediate families (other than students) are ineligible for these prizes; competition is open to everyone else. All decisions by the judges are final. Multiple entries are allowed.

1.1. Victoria Delfino. Victoria Delfino was a realtor in the Los Angeles area who helped Yiannis Moschovakis buy his house. ${ }^{1}$ When Tony Martin moved to UCLA, Moschovakis referred him to Delfino, who also became Martin's realtor and found the house where Martin still lives. Two weeks after the sale was finalised, Delfino gave Moschovakis an amount of money as commission for the referral, and did not accept his attempts to reject it.

As a result, Moschovakis decided instead to use the money to help fund the series of Very Informal Gatherings, the first of which had taken place in

The first author thanks the National Science Foundation for partial support through grant DMS-0801189.

${ }^{1}$ Most of this section is based on recollections shared by Moschovakis with the first author during a telephone conversation. 
the fall of $1975 .^{2}$ The second Very Informal Gathering, in 1978, started a new tradition: with a single exception, all subsequent VIGs have taken place on Super Bowl weekend, in late January or early February. Moschovakis comments:

The time of the only exception, there was an earthquake! A clear sign that moving the date was a mistake.

Together with the funding of the Very Informal Gatherings, the money was also set to cover the prizes for the solutions of the five original Delfino Problems. (Contrary to popular belief, no monetary prize was attached to further problems.)

When Moschovakis introduced these five problems (in what he described as one of the most significant Very Informal Gatherings to date), and mentioned the Victoria Delfino Fund, Martin, taken by surprise, exclaimed "That's my broker!" Not all in attendance heard this, and Moschovakis offered no further explanation for the name of the fund. This led for a short while to a variety of conjectures trying to find appropriate interpretations to explain the name.

Originally, the fund was kept in a joint account by Alexander Kechris, Martin, and Moschovakis. It was supplemented by occasional donations from other logicians in the area. Martin reports (personal communication) that by 1998, "all the money in the fund had been used and we had stopped asking people to contribute to it." Eventually, it became so low that it made sense to use it all and close the account. Nowadays, the Very Informal Gatherings are typically funded through support of the NSF.

As for Delfino, she eventually retired, moved out of state to take care of an ill relative, and her trail disappears there. It is unknown whether she ever found out that her name was associated with the problems or with the Cabal.

1.2. The problems. After the first announcement of the Victoria Delfino Problems, progress reports were published in [CABAL ii] and [CABAL iii]. In 1985 , three of the original problems had been solved, and seven new problems (\# 6 to \# 12) were added and published as [KMS88A] in [CABAL iv], preceded by the following comment:

At the "Very Informal Gathering" of January 1984, the Cabal announced the addition of seven problems to the Victoria Delfino list. We are happy (and not at all embarrassed) to report that since then four of these problems have been solved. Below we list the new problems, beginning with \# 6 since there were five problems on the original list. For each we describe briefly what was known when it was added to the list, and what has been its fate since.

\footnotetext{
${ }^{2}$ There is some uncertainty about the date of the first VIG; in preparation for the twentieth VIG in February 2019, the original organisers discussed this question and concluded that "our best recollection now is that the first VIG was in the fall of 1975" (Kechris, personal communication, 2018).
} 
In the years following the publication of the final original Cabal volume, there were two more problems announced at one of the Very Informal Gatherings in the late 1980s or early 1990s (the precise date could not be identified), but they were never published as Victoria Delfino problems. We include these problems as \# 13 and \# 14.

Today, two of the problems remain open. The first one is better known under the name of Martin's Conjecture (\# 5), the other one has now been embedded into Woodin's theory $\mathrm{AD}^{+}(\# 14)$. In Table 1, the reader can find a synoptic list of the problems with their current status.

\begin{tabular}{|c|c|c|}
\hline & Published in & Status \\
\hline$\# 1$ & [KM78A] & Solved by Steve Jackson (1983) \\
\hline \#2 & [KM78A] & Solved by Yiannis Moschovakis (1981) \\
\hline \# 3 & [KM78A] & Solved by Howard Becker \& Alexander Kechris (1983) \\
\hline \#4 & [KM78A] & Solved by John Steel (1993) \\
\hline \# 5 & [KM78A] & Open \\
\hline \#6 & [KMS88A] & Solved by John Steel (1984) \\
\hline \# 7 & [KMS88A] & Solved by Steve Jackson (1985) \\
\hline \# 8 & [KMS88A] & Solved by John Steel (1994) \\
\hline \#9 & [KMS88A] & Solved by Tony Martin \& John Steel (1985) \\
\hline \# 10 & [KMS88A] & Solved by W. Hugh Woodin \& Saharon Shelah (1985) \\
\hline \# 11 & [KMS88A] & Solved by John Steel (1994) \\
\hline \# 12 & [KMS88A] & Solved by John Steel (1997) \\
\hline \#13 & unpublished & Solved by W. Hugh Woodin (1999) \\
\hline \# 14 & unpublished & Open \\
\hline
\end{tabular}

TABLE 1. List of the Victoria Delfino problems and their current status

This paper is organised as follows: Each problem is presented in its own section that, except for the last two problems, starts with a quote from the original Cabal volumes under the headline Original problem. The quotation is essentially literal, although we have followed the general practice of modernising and homogenising notation and writing style. For the first five problems, the original formulation is followed by one or several subsections entitled Progress report where we reproduce the text from subsequent Cabal volumes providing updates on the problem. We then proceed with a brief discussion of the current state of knowledge in a subsection entitled 2020 comments.

1.3. Acknowledgements. We should like to thank Kai Hauser, Daisuke Ikegami, Antonio Montalbán, Jan Reimann, Ralf Schindler, Ted Slaman, John Steel, Simon Thomas, Hugh Woodin, and Yizheng Zhu for detailed remarks and comments. Particular thanks are due to Yiannis Moschovakis and Tony 
Martin for their recollections on Victoria Delfino, the Delfino Fund, and the early history of the Very Informal Gatherings.

\section{\# 1. Projective Ordinals.}

Original problem [KM78A]. For each positive integer $n$, let $\boldsymbol{\delta}_{n}^{1}$ be the least nonzero ordinal not the length of a $\Delta_{n}^{1}$ prewellordering of the reals. Assume AD + DC. It is known that ${\underset{\sim}{1}}_{1}^{1}=\omega_{1},{\underset{\sim}{2}}_{2}^{1}=\omega_{2},{\underset{\sim}{3}}_{3}^{1}=\omega_{\omega+1},{\underset{\boldsymbol{\delta}}{4}}_{4}^{1}=\omega_{\omega+2}$, ${\underset{\boldsymbol{\delta}}{2 n+2}}_{1}^{1}=\left(\boldsymbol{\delta}_{2 n+1}^{1}\right)^{+}$, and $\boldsymbol{\delta}_{2 n+1}^{1}$ is always the successor (cardinal) of a cardinal of cofinality $\omega$.

\section{Problem \# 1. Compute $\boldsymbol{\delta}_{5}^{1}$.}

Kunen has some partial results on this problem, results which suggest the answer $\omega_{\omega^{3}+1}$.

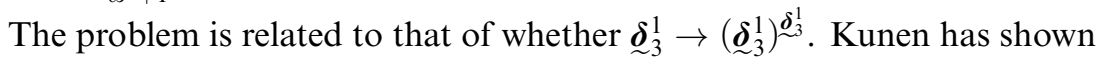
that ${\underset{\sim}{3}}_{3}^{1} \rightarrow(\underbrace{1}_{3})^{\alpha}$ for each $\alpha<\underline{\boldsymbol{\delta}}_{3}^{1}$. Results of Kleinberg imply that ${\underset{\sim}{3}}_{3}^{1}$ has exactly three normal measures. It is likely that the regular cardinals between $\boldsymbol{\delta}_{3}^{1}$ and ${\underset{\sim}{5}}_{5}^{1}$ are exactly the ultrapowers of $\boldsymbol{\delta}_{3}^{1}$ with respect to these normal measures. This would be important in getting an upper bound on $\boldsymbol{\delta}_{5}^{1}$ from Choice plus $A D^{\mathbf{L}(\mathbb{R})}$, the hypothesis that every set of reals in $\mathbf{L}(\mathbb{R})$ is determined.

(Needless to say, the decision of the judges as to what constitutes a "computation" of $\boldsymbol{\delta}_{5}^{1}$ will be final.)

Progress report [KMM81A]. Martin has established the conjectured lower bound for $\boldsymbol{\delta}_{5}^{1}$ by proving (from AD + DC) that

$$
\stackrel{\delta}{5}_{5}^{1} \geq \aleph_{\omega^{3}+1} ;
$$

moreover Martin showed (from AD) that the ultrapowers of $\boldsymbol{\delta}_{3}^{1}=\aleph_{\omega+1}$ under the three normal measures on ${\underset{\sim}{3}}_{3}^{1}$ are exactly $\boldsymbol{\delta}_{4}^{1}=\aleph_{\omega+2}$ (this was known to Kunen), $\aleph_{\omega \cdot 2+1}$ and $\aleph_{\omega^{2}+1}$ and that these three cardinals are measurable (and hence regular), so that (in particular), $\boldsymbol{\delta}_{5}^{1}$ is not the first regular cardinal after $\boldsymbol{\delta}_{4}^{1}$. We still have no upper bounds for ${\underset{\boldsymbol{\delta}}{5}}_{5}^{1}$ from AD.

Progress report [KMM83A]. It was announced in [KMM81A] that Martin had shown ${\underset{\sim}{\delta}}_{5}^{1} \geq \aleph_{\omega^{3}+1}$ and that the ultrapowers of $\boldsymbol{\delta}_{3}^{1}$ with respect to the three normal measures on $\boldsymbol{\delta}_{3}^{1}$ are $\aleph_{\omega+2}, \aleph_{\omega \cdot 2+1}$ and $\aleph_{\omega^{2}+1}$. The proof of part of the last assertion, that the ultrapower by the $\omega_{2}$-cofinal measure is $\leq \aleph_{\omega^{2}+1}$, was incorrect. Actually this ultrapower is larger $\left(\aleph_{\omega^{\omega}+1}\right)$.

Steve Jackson has completely solved the first problem. He first proved that $\boldsymbol{\delta}_{5}^{1} \leq \aleph_{\omega^{\left(\omega^{(\omega)}\right)}+1}$. This result will appear in his UCLA Ph.D. Thesis. He next used the machinery for getting this upper bound to analyze all measures on $\boldsymbol{\delta}_{3}^{1}$ and to get a good representation of functions with respect to these measures. Martin observed that this representation and ideas of Kunen allow one to show 
${\underset{\boldsymbol{\delta}}{\boldsymbol{\delta}}}_{3}^{1} \rightarrow\left(\underline{\boldsymbol{\delta}}_{3}^{1}\right)^{\delta_{3}^{1}}$. From this it follows by a result of Martin that the ultrapower of $\boldsymbol{\delta}_{3}^{1}$ with respect to any of its measures is a cardinal. Jackson's analysis then gives $\boldsymbol{\delta}_{5}^{1} \geq \aleph_{\omega^{\left(\omega^{(\omega)}+1\right.}}$ so ${\underset{\sim}{\delta}}_{5}^{1}=\aleph_{\omega^{\left(\omega^{(\omega)}+1\right.}}$.

2020 comments. Steve Jackson not only solved Problem \# 1, but also solved the problem in general for all projective ordinals. He computed $\boldsymbol{\delta}_{2 n+1}^{1}$ to be $\aleph_{\mathbf{e}_{n}+1}$ where $\mathbf{e}_{0}:=0$ and $\mathbf{e}_{i+1}:=\omega^{\left(\omega^{\mathbf{e}_{i}}\right)}$ (i.e., $\mathbf{e}_{n}$ is an exponential $\omega$-tower of height $2 n-1$ ).

However, Jackson's paper [Jac88] where the inequality $\boldsymbol{\delta}_{2 n+1}^{1} \leq \aleph_{\mathbf{e}_{n}+1}$ is established, is notoriously hard to read, and so in the decades following his solution of the problem, Jackson produced various expositions of the results. As the title "A computation of $\boldsymbol{\delta}_{5}^{1}$ " suggests, his book [Jac99] focuses on the (complete) computation of $\boldsymbol{\delta}_{5}^{1}$ as asked in the original problem, and explains how to proceed to compute all projective ordinals via an inductive analysis. His survey paper [Jac10] also discusses extensions of these results beyond the projective ordinals:

In the early 1980s, Martin [Mar] obtained a result on the ultrapowers of $\boldsymbol{\delta}_{3}^{1}$ by the normal measures on $\boldsymbol{\delta}_{3}^{1}$. Building on this and some joint work with Martin, [Jackson] computed $\boldsymbol{\delta}_{5}^{1}$. In the mid-1980s, this was extended to compute all the $\boldsymbol{\delta}_{n}^{1}$, and to develop the combinatorics of the cardinal structure of the cardinals up to that point. The analysis, naturally, proceeded by induction. The complete "firststep" of the induction appears in [Jac99]. The analysis revealed a rich combinatorial structure to these cardinals. [ ... ] A goal, then, is to extend some version of this "very-fine" structure theory to the entire model $\mathbf{L}(\mathbb{R})$. In the late 1980s, [Jackson] extended the analysis further, up to the least inaccessible cardinal in $\mathbf{L}(\mathbb{R})$, although this lengthy analysis has never been written up. It was clear, however, that new, serious problems were being encountered shortly past the least inaccessible. In [Jac91], for example, results were given that show that the theory fell far short of $\kappa^{\mathbb{R}}$, the ordinal of the inductive sets (the Wadge ordinal of the least non-selfdual pointclass closed under real quantification). [Jac10, p. 1755]

Part of the extended results is what is known as Kechris's theorem:

THeOREM 1. Assume $\mathrm{AD}+\mathbf{V}=\mathbf{L}(\mathbb{R})$. If $\kappa$ is an inaccessible Suslin cardinal, ${ }^{3}$ then $\kappa, \kappa^{+}$, and $\kappa^{++}$are measurable.

Kechris's theorem remained unpublished for many years; a proof and generalisations to polarised partition properties for $\kappa, \kappa^{+}$, and $\kappa^{++}$can be found in [AJL13].

\footnotetext{
${ }^{3}$ E.g., the Kleene ordinal discussed in Problem $\# 7$ or $\kappa^{\mathbb{R}}$, the least non-hyperprojective ordinal; $c f$. [AJL13, Proposition 5].
} 
Further projects to make the proof and its generalisations more accessible are what Jackson calls "description theory" (cf. [JK16]) and the "simple inductive arguments" given in [Löw02, BL07, Bo109]. On the basis of the inductive analysis, Jackson and the second author developed an abstract theory of canonical measure assignments that allows blackboxing the proofs of partition properties and deriving consequences (such as the behaviour of the cofinality function or the calculation of the measurable cardinals) directly by induction (cf. [JL13]).

The ZFC context. At the end of the original formulation of Problem \# 1, the question of calculating bounds for the projective ordinals in $Z F C+A D^{L(R)}$ is mentioned. The original AD-results listed in the original formulation of Problem \# 1 yield upper bounds in this context: ${\underset{\sim}{1}}_{1}^{1}=\aleph_{1},{\underset{\boldsymbol{\delta}}{1}}_{2}^{1} \leq \aleph_{2}, \underline{\boldsymbol{\delta}}_{3}^{1} \leq \aleph_{3}$, $\tilde{\boldsymbol{\delta}}_{4}^{1} \leq \aleph_{4}, \boldsymbol{\delta}_{5}^{1} \leq \aleph_{7}$. In general, Jackson's analysis shows that under AD, there are exactly $2^{n}-1$ regular cardinals below ${\underset{\delta}{2 n+1}}_{2}^{1}$; thus $Z F C+\mathrm{AD}^{\mathbf{L}(\mathbb{R})}$ proves that $\boldsymbol{\delta}_{n}^{1}<\aleph_{\omega}$ for every natural number $n$. Martin conjectured that "for all $n,{\underset{\delta}{n}}_{n}^{1}=\aleph_{n}$ ” should follow from ZFC $+\mathrm{AD}^{\mathbf{L}(\mathbb{R})}$ plus reasonable additional assumptions (cf., e.g., [Woo99, p. 5]).

In [Woo99], Woodin develops a very powerful technique to produce models of ZFC as forcing extensions of models of determinacy, the analysis of which provides a solution to Martin's conjecture in the case $n=2$ :

THeorem 2 (Woodin; [Woo99, Theorem $1.1 \& \S 3.1$ ]). If the nonstationary ideal on $\omega_{1}$ is $\omega_{2}$-saturated and $\wp\left(\omega_{1}\right)^{\#}$ exists, then $\boldsymbol{\delta}_{2}^{1}=\aleph_{2}$.

On the other hand, Woodin points out that current techniques produce models where $\boldsymbol{\delta}_{3}^{1}<\Theta^{\mathbf{L}(\mathbb{R})}=\aleph_{3}$, and asks-in contrast to Martin's conjecturewhether it is a theorem of ZFC $+A D^{\mathbf{L}(\mathbb{R})}$ that $\Theta^{\mathbf{L}(\mathbb{R})} \leq \aleph_{3}[$ Woo99, $\S 1.5]$. Martin's conjecture for $n>2$ and the competing question by Woodin remain open.

\section{\# 2. The extent of definable scales.}

Original problem [KM78A]. A semiscale on a set $P \subseteq \mathbb{R}^{k}\left(\mathbb{R}={ }^{\omega} \omega\right)$ is a sequence $\vec{\varphi}=\left\{\varphi_{n}: n \in \omega\right\}$ of norms on $P$, where each $\varphi_{n}: P \rightarrow \lambda$ maps $P$ into some ordinal $\lambda$ and the following convergence condition holds: If $x_{0}, x_{1}, \cdots \in P$ and for each $n$ the sequence $\varphi_{n}\left(x_{0}\right), \varphi_{n}\left(x_{1}\right), \varphi_{n}\left(x_{2}\right), \ldots$ is ultimately constant, then $x \in P$. We call $\vec{\varphi}$ a scale if, under the same hypotheses, we can infer that

$$
\varphi_{n}(x) \leq \varphi_{n}\left(x_{i}\right) \text { for all large } i .
$$

A semiscale $\vec{\varphi}$ is in a class of relations $\Gamma$ if both relations

$$
\begin{aligned}
& U(n, x, y) \Longleftrightarrow x \in P \wedge\left[y \notin P \vee \varphi_{n}(x) \leq \varphi_{n}(y)\right] \\
& V(n, x, y) \Longleftrightarrow x \in P \wedge\left[y \notin P \vee \varphi_{n}(x)<\varphi_{n}(y)\right]
\end{aligned}
$$

are in $\Gamma$. 
It is easy to check that a set $P$ admits a semiscale $\vec{\varphi}$ into $\lambda$ if and only if $P$ is $\lambda$-Suslin, i.e., $P$ is the projection of some tree $T$ on $\omega^{k} \times \lambda$; moreover, $T$ is definable exactly when $\vec{\varphi}$ is definable. Sets which admit definable scales are well-behaved in many ways, e.g. we can use a scale on $P \subseteq \mathbb{R} \times \mathbb{R}$ to uniformise $P$.

Granting projective determinacy, we can prove that every projective set admits a projective scale (Moschovakis); on the other hand it is easy to check that $\{(x, y): x$ is not ordinal definable from $y\}$ does not admit a scale which is OD in a real, granting only that for each $y$ there is some $x$ which is not OD in $y$. Thus not every "definable" set admits a "definable" scale.

The strongest result we can get with current methods is that inductive sets admit inductive scales, granting inductive determinacy; here $P$ is inductive if $P$ is $\Sigma_{1}$ over the smallest admissible set $M$ which contains the reals, $\mathbb{R} \in M$.

Problem \#2. Assume $\mathrm{ZF}+\mathrm{DC}+\mathrm{AD}+\mathbf{V}=\mathbf{L}(\mathbb{R})$; prove or disprove that every coinductive set of reals is $\lambda$-Suslin for some $\lambda$.

Progress report [KMM81A]. The problem was solved by Moschovakis who showed (from $A D+D C$ ) that every coinductive pointset admits a scale. If we let $\Sigma_{0}^{*}$ be the set of all Boolean combinations of inductive and coinductive sets and then define $\Sigma_{n}^{*}$ by counting quantifiers over $\mathbb{R}$ in front of a $\Sigma_{0}^{*}$ matrix in the usual way, then the proof shows that every coinductive set admits a scale $\left\{\varphi_{n}: n \in \omega\right\}$, where each $\varphi_{n}$ is a $\Sigma_{n+1}^{*}$-norm, uniformly in $n$.

Martin and Steel extended the method used by Moschovakis in this proof and showed that $Z F+D C+A D+V=\mathbf{L}(\mathbb{R})$ implies that every $\Sigma_{1}^{2}$ set admits a $\Sigma_{1}^{2}$-scale; this combines with an earlier result of Kechris and Solovay to show that $Z F+D C+A D+\mathbf{V}=\mathbf{L}(\mathbb{R})$ implies that a pointset admits a scale if and only if it is $\underset{\sim}{\Sigma_{1}^{2}}$.

Martin then combined these ideas with the technique of the Third Periodicity Theorem [Mos80, Theorem 6E.1] and showed that under reasonable hypotheses of determinacy for games on $\mathbb{R}$, (namely, $A D_{\mathbb{R}}$ ), the scale property is preserved by the game quantifier $5^{2}$ on $\mathbb{R}$, where

$$
\left(\supset^{2} \alpha\right) P(x, \alpha) \Longleftrightarrow\left(\exists \alpha_{0}\right)\left(\forall \alpha_{1}\right)\left(\exists \alpha_{2}\right)\left(\forall \alpha_{3}\right) \ldots P\left(x,\left\langle\alpha_{0}, \alpha_{1}, \ldots\right\rangle\right) .
$$

This result produces scales for sets that are not ${\underset{\sim}{\sim}}_{1}^{2}$ in $\mathbf{L}(\mathbb{R})$ and leaves open the general question of the extent of scales in the presence of axioms stronger than AD.

2020 comments. Moschovakis's paper appeared as [Mos83]. The result of Martin and Steel appears in [MS83]. Martin's theorem on preservation of scales under the game quantifier $\mathrm{J}^{2}$ is in [Mar83]. Related results by Steel are in [Ste83B] and [Ste83A]. The latter paper introduces the key fine structural analysis of $\mathbf{L}(\mathbb{R})$ via gaps that is now used in the core model induction. These results have been further extended by Steel (under appropriate large cardinals 
or stronger determinacy assumptions), $c f$., e.g., [Ste08E, Ste08D], and the introduction [Ste08B]. These extensions are needed for core model inductions whose goal is to reach models of strong determinacy assumptions.

\section{\# 3. The invariance of $\mathrm{L}\left[T^{3}\right]$.}

Original problem [KM78A]. Let $n$ be an odd integer. Let $P$ be a complete $\Pi_{n}^{1}$ set of reals and assuming PD let $\vec{\varphi}=\left\{\varphi_{m}: m \in \omega\right\}$ be a $\Pi_{n}^{1}$-scale on $P$. (It is understood here that each $\varphi_{m}$ maps $P$ onto an initial segment of the ordinals.) The tree $T^{n}=T^{n}(\vec{\varphi})$ associated with this scale is defined by

$$
T^{n}=\left\{\left\langle\alpha(0), \varphi_{0}(\alpha), \ldots, \alpha(k), \varphi_{k}(\alpha)\right\rangle: \alpha \in P\right\} .
$$

Let $A D^{\mathbf{L}(\mathbb{R})}$ be the hypothesis that every set of reals in $\mathbf{L}(\mathbb{R})$ is determined.

Problem \#3. Assume $\mathrm{ZF}+\mathrm{DC}+\mathrm{AD}^{\mathbf{L}(\mathbb{R})}$. Prove or disprove that $\mathbf{L}\left[T^{3}\right]=$ $\mathbf{L}\left[T^{3}(\vec{\varphi})\right]$ is independent of the choice of the complete $\Pi_{3}^{1}$ set $P$ and the particular $\Pi_{3}^{1}$-scale $\vec{\varphi}$ on $P$.

It is known that $\mathbf{L}\left[T^{1}\right]=\mathbf{L}$ (Moschovakis). Also under the above hypothesis it is known that for all odd $n$ and all $T^{n}=T^{n}(\vec{\varphi}), \mathbf{L}\left[T^{n}\right] \cap \mathbb{R}=\mathrm{C}_{n+1}$, where $\mathrm{C}_{n+1}$ is the largest countable $\Sigma_{n+1}^{1}$ set of reals (Harrington-Kechris), so that $\mathbb{R} \cap \mathbf{L}\left[T^{n}\right]$ does not depend on the choice of $T^{n}$.

In many ways, the model $\mathbf{L}\left[T^{n}\right]$ is an excellent analog of $\mathbf{L}$ for the $(n+1)$-st level of the analytical hierarchy.

Progress report [KMM81A]. Kechris showed in [Kec81] that if $T^{3}=T^{3}(\vec{\varphi})$ is the tree associated with some $\Pi_{3}^{1}$-scale $\vec{\varphi}$ on a $\Pi_{3}^{1}$-complete set $P$ and if

$$
\widetilde{\mathbf{L}}\left[T^{3}\right]=\bigcup_{\alpha \in \mathbb{R}} \mathbf{L}\left[T^{3}, \alpha\right],
$$

then $\mathrm{ZF}+\mathrm{AD}+\mathrm{DC}+{\underset{\boldsymbol{\delta}}{3}}_{3}^{1} \rightarrow\left({\underset{\sim}{3}}_{3}^{1}\right)^{\boldsymbol{\delta}_{3}^{1}}$ implies that $\widetilde{\mathbf{L}}\left[T^{3}\right]$ is independent of the choice of $P$ and $\vec{\varphi}$.

This partial result emphasises the importance of the question of the strong partition property for $\boldsymbol{\delta}_{3}^{1}$ which is still open.

Progress report [KMM83A]. The problem was solved by Becker and Kechris who showed that $L\left[T^{3}\right]$ is independent of the choice of $T^{3}$. This is a consequence of the following fact, which is a theorem of ZF + DC.

THEOREM 3. Let $\Gamma$ be an $\omega$-parametrised pointclass closed under $\wedge$ and recursive substitution and containing all recursive sets. Let $P \subset \mathbb{R}$ be a complete $\Gamma$ set, $\vec{\varphi}=\left\{\varphi_{i}: i \in \omega\right\}$ be an $\exists^{\mathbb{R}} \Gamma$-scale on $P$ such that all norms $\varphi_{i}$ are regular, and $\kappa=\sup \left\{\varphi_{i}(x): i \in \omega, x \in P\right\}$. Let $T(\vec{\varphi})$ be the tree on $\omega \times \kappa$ associated with $\vec{\varphi}$. For any set $A \subset \kappa$, if $A$ is $\exists^{\mathbb{R}} \Gamma$-in-the-codes with respect to $\vec{\varphi}$ (that is, if the set $\left\{\langle i, x\rangle \in \omega \times \mathbb{R}: x \in P \wedge \varphi_{i}(x) \in A\right\}$ is $\left.\exists^{\mathbb{R}} \Gamma\right)$, then $A \in \mathbf{L}[T(\vec{\varphi})]$. 
In general, given two such scales $\vec{\varphi}, \vec{\psi}$, it is not known that $T(\vec{\psi})$ is $\exists^{\mathbb{R}} \Gamma$-inthe-codes with respect to $\vec{\varphi}$, so the invariance of $\mathbf{L}[T(\vec{\varphi})]$ has not been shown in this generality. However there are special cases where invariance can be proved. Henceforth, assume AD.

In Moschovakis [Mos80, p. 562], a model $H_{\Gamma}$ is defined for every pointclass $\Gamma$ which resembles $\Pi_{1}^{1}$; this includes the pointclasses $\Pi_{n}^{1}$ for odd $n$. It follows from Theorem 3, together with known results about the $H_{\Gamma}$ 's [Mos80, 8G], that for any $\Gamma, P, \vec{\varphi}$ such that $\Gamma$ resembles $\Pi_{1}^{1}$ and $\Gamma, P, \vec{\varphi}$ satisfy the assumptions of Theorem $3, L[T(\vec{\varphi})]=H_{\Gamma}$, and hence $\mathbf{L}[T(\vec{\varphi})]$ is independent of the choice of $P$ and $\vec{\varphi}$. For $\Gamma=\Pi_{3}^{1}$ this solves the third problem.

While the invariance problem for $\mathbf{L}\left[T^{n}\right]$ is thus solved for odd $n$, for even $n$ the situation is still unclear. Call a $\Sigma_{3}^{1}$-scale on a $\Pi_{2}^{1}$ set good if it satisfies the ordinal quantification property of Kechris-Martin [KM78]. It follows from the above theorem that $\mathbf{L}\left[T^{2}\right]$ is independent of the choice of a complete $\Pi_{2}^{1}$ set and of the choice of a good scale. Whether or not it is independent of the choice of an arbitrary scale is unknown. For even $n>2$, it is not known whether there exist any good scales.

2020 comments. The result by Becker and Kechris for odd $n$ appears in [BK84]. In [Hjo96B], Hjorth shows that, under $\operatorname{Det}(\underset{\sim}{\mathbf{\Pi}} \mathbf{1})$, the model $\mathbf{L}\left[T^{2}\right]$ is independent of the exact choice of $T^{2}$. His argument uses forcing to analyze $\Pi_{3}^{1}$ equivalence relations. In [Hjo95], he uses properties of $\mathbf{L}\left[T^{2}\right]$ to draw descriptive set theoretic consequences of the assumption that all reals have sharps, in particular showing that if all reals have sharps and $\mathrm{MA}_{\omega_{1}}$ holds, then all $\Sigma_{3}^{1}$ sets are Lebesgue measurable. Further work on $\mathbf{L}\left[T^{2}\right]$ using fine-structural techniques has been carried out by Hauser [Hau99].

In [Atm19], Atmai shows that $\mathbf{L}\left[T^{2 n}\right]$ is independent of the choice of $T^{2 n}$, assuming $\operatorname{Det}\left({\underset{\sim}{2 n}}_{2 n}^{1}\right)$. His proof involves an appropriate generalisation of the Kechris-Martin theorem to the odd levels of the projective hierarchy. Atmai also shows that the $\mathbf{L}\left[T^{2 n}\right]$ are not extender models, but satisfy some of their properties, such as $\mathrm{GCH}$.

Meanwhile, developments in inner model theory have provided us both with new methods for analyzing the models $\mathbf{L}\left[T^{n}\right]$, and with the proper analogues of $\mathbf{L}$ for higher levels of the analytic hierarchy, the fine structural models $\mathbf{M}_{n}$. Recall that (under appropriate large cardinal assumptions) $\mathbf{M}_{n}$ is the canonical minimal inner model for the assumption that there are $n$ Woodin cardinals. In [Ste95B], Steel gives a precise definition of $\mathbf{M}_{n}$ in terms of $n$-smallness and shows that $\mathbf{M}_{n}$ is $\Sigma_{n}^{1}$-correct, and that $\mathbb{R} \cap \mathbf{M}_{n}=\mathrm{C}_{n}$ for $n$ even, and $\mathbb{R} \cap \mathbf{M}_{n}=\mathrm{Q}_{n}$ for odd $n$. For odd $n$, it is unknown whether the sets $\mathrm{C}_{n}$ in general have an inner model theoretic characterisation. For $n=1$, Guaspari, Kechris, and Sacks independently showed that $\mathrm{C}_{1}=\left\{x \in \mathbb{R}: x \in \mathbf{L}_{\omega_{1}^{x}}\right\}$ [Gua73, Kec75, Sac76]. For $k>0$, the analogous statement " $\mathrm{C}_{2 k+1}$ is the set of reals $\Delta_{2 k+1}^{1}$-equivalent to the first order theory of some level of $\mathbf{M}_{2 k}$ 
projecting to $\omega$ " is open and known as the $\mathrm{C}_{3}$ conjecture [Ste08B, p. 13] (cf. also [GH76, Cra85, Zhu17]).

\section{\# 4. The strength of $\operatorname{Sep}\left(\Sigma_{3}^{1}\right)$ in the presence of sharps.}

Original problem $[\mathrm{KM} 78 \mathrm{~A}]$. Let $(\#)$ stand for "for all $x \subseteq \omega, x^{\#}$ exists" and let $\operatorname{Sep}\left(\Sigma_{3}^{1}\right)$ denote "for every $x \subseteq \omega$, every two disjoint $\Sigma_{3}^{1}(x)$ sets of reals can be separated by a $\Delta_{3}^{1}(x)$ set."

Problem \# 4. Prove or disprove that

$$
\mathrm{ZFC}+\operatorname{Sep}\left(\Sigma_{3}^{1}\right)+(\#) \text { implies } \operatorname{Det}\left(\boldsymbol{\Delta}_{2}^{1}\right) \text {. }
$$

Harrington has shown that $\mathrm{ZFC}+\operatorname{Sep}\left(\Sigma_{3}^{1}\right)$ is consistent relative to ZFC. However, using Jensen's Absoluteness Theorem for the core model $\mathbf{K}$ (which states that if (\#) holds and $\Sigma_{3}^{1}$ formulas are not absolute for $\mathbf{K}$, then $0^{\dagger}$ exists) one can see that

$$
\mathrm{ZF}+\mathrm{DC}+\operatorname{Sep}\left(\Sigma_{3}^{1}\right)+(\#) \text { implies that } x^{\dagger} \text { exists for all } x \subseteq \omega .
$$

2020 comments. Problem \# 4 was solved with core model techniques by John Steel, following the approach mentioned in the last paragraph of the original problem. The result appears as [Ste96, Corollary 7.14]. The key result is that if there are no inner models with Woodin cardinals and there exists a measurable cardinal, then $\mathbf{K}$ is $\Sigma_{3}^{1}$-correct [Ste96, Theorem 7.9]. In the setting of that book, an additional larger measurable $\Omega$ is assumed in the background and a set sized $\mathbf{K}$ is built of height $\Omega$; this additional assumption is now known not to be necessary; cf. [JS13].

To solve Problem \# 4 affirmatively, Steel argues that $\operatorname{Sep}\left(\Sigma_{3}^{1}\right)+(\#)$ implies that for every real $x$ there is a proper class model $M$ with $x \in M$, and an ordinal $\delta$ such that $\mathbf{V}_{\delta+1}^{M}$ is countable, and $\delta$ is Woodin in $M$. By results of Woodin, this implies $\operatorname{Det}\left(\Delta_{2}^{1}\right)$ ( $c f$. the 2020 comments on Problem \# 9 and [Nee10, Corollary 6.12]). To see that such a model $M$ exists, one first uses the core model argument mentioned in the original problem: for any real $y$, the $\Sigma_{3}^{1}$-correctness of the Mitchell core model gives a proper class model $N$ with $y \in N$ and two measurable cardinals. ${ }^{4}$

Once we have $N$, Steel argues that if $y$ is chosen carefully to ensure that $\operatorname{Sep}\left(\Sigma_{3}^{1}\right)$ relativises down from $\mathbf{V}$ to $N$, the $\mathbf{K}_{x}$ construction inside $N$ must fail: Assuming that $\left(\mathbf{K}_{x}\right)^{N}$ exists, then it is $\Sigma_{3}^{1}$-correct inside $N$. But there is a $\Delta_{3}^{1}(x)$ well-ordering of the reals of $\left(\mathbf{K}_{x}\right)^{N}$, which implies the failure of $\operatorname{Sep}\left(\Sigma_{3}^{1}\right)$ inside $\left(\mathbf{K}_{x}\right)^{N}$. But by our choice of $y, \operatorname{Sep}\left(\Sigma_{3}^{1}\right)$ relativises down from $\mathbf{V}$ to $N$ and the correctness of $\mathbf{K}_{x}$ inside $N$ implies that it further relativises down from $N$ to $\left(\mathbf{K}_{x}\right)^{N}$, which is impossible.

\footnotetext{
${ }^{4}$ If we make use of [JS13], we only need one measurable cardinal and could use the Dodd-Jensen core model here.
} 
Thus, the $\mathbf{K}_{x}$ construction inside $N$ fails and and therefore $\left(\mathbf{K}_{x}^{\mathrm{c}}\right)^{N}$ reaches a Woodin cardinal, and an iterate of an appropriate hull of $\left(\mathbf{K}_{x}^{\mathrm{c}}\right)^{N}$ is the model $M$ as needed.

It is still open whether there is a $\Sigma_{3}^{1}$-correctness theorem for $\mathbf{K}$ (in the absence of Woodin cardinals) without additional assumptions beyond the existence of sharps.

\section{\# 5. A classification of functions on the Turing degrees.}

Original problem [KM78A]. We write $\mathcal{D}$ for the set of Turing degrees. A property $P$ of degrees holds almost everywhere (a.e.) if and only if there is a $\mathbf{c}$ such that for all $\mathbf{d} \geq \mathbf{c}$, we have $P(\mathbf{d})$. For $f, g: \mathcal{D} \rightarrow \mathcal{D}$, let $f \leq_{\mathrm{m}} g$ if and only if $f(\mathbf{d}) \leq g(\mathbf{d})$ a.e. A function $f: \mathcal{D} \rightarrow \mathcal{D}$ is representable if and only if there is some $F:{ }^{\omega} \omega \rightarrow{ }^{\omega} \omega$ such that for all $x, \operatorname{deg}(F(x))=f(\operatorname{deg}(x))$.

Problem \# 5. Working in ZF $+\mathrm{AD}+\mathrm{DC}$, settle the following conjectures of D. Martin:

(a) If $f: \mathcal{D} \rightarrow \mathcal{D}$ is representable and $\mathbf{d} \not \leq f(\mathbf{d})$ a.e., then there is a $\mathbf{c}$ such that $f(\mathbf{d})=\mathbf{c}$ a.e.

(b) The relation $\leq_{\mathrm{m}}$ is a prewellorder of $\{f: f$ is representable and $\mathbf{d} \leq f(\mathbf{d})$ a.e.\}.

Further, if $f$ has rank $\alpha$ in $\leq_{\mathrm{m}}$, then $f^{\prime}$ has rank $\alpha+1$, where $f^{\prime}(\mathbf{d})=f(\mathbf{d})^{\prime}$, the Turing jump of $f(\mathbf{d})$.

Remarks. With regard to (a), it is well known that if $f(\mathbf{d}) \leq \mathbf{d}$ and $\forall \mathbf{c}(\mathbf{c} \leq f(\mathbf{d})$ a.e. $)$, then $f(\mathbf{d})=\mathbf{d}$ a.e. It is known that conjecture (b) is true when restricted to uniformly representable $f$ so that $\mathbf{d} \leq f(\mathbf{d})$ a.e. (A function $f$ is uniformly representable if there is an $F:{ }^{\omega} \omega \rightarrow{ }^{\omega} \omega$ such that for all $x$, we have $\operatorname{deg}(F(x))=f(\operatorname{deg}(x))$ and, moreover, there is a $t: \omega \rightarrow \omega$ such that for all $x$ and $y$, if $x \equiv_{\mathrm{T}} y$ via $e$ then $F(x) \equiv_{\mathrm{T}} F(y)$ via $t(e)$.) It is conjectured that every representable $f: \mathcal{D} \rightarrow \mathcal{D}$ is uniformly representable.

A proof of conjecture (b) would yield a strong negative answer to a question of Sacks: is there a degree invariant solution to Post's problem?

Progress report [KMM81A]. It follows from unpublished results of Kechris and Solovay that $Z F+A D+D C+\mathbf{V}=\mathbf{L}(\mathbb{R})$ implies that every function $f: \mathcal{D} \rightarrow \mathcal{D}$ on the degrees is representable. Although this has no direct bearing on a possible solution of the fifth problem, it underscores the generality of the question.

Progress report [KMM83A]. Slaman and Steel have proved two theorems relevant to Problem \# 5. The first verifies a special case of conjecture (a):

Theorem 4. ( $\mathrm{ZF}+\mathrm{AD}+\mathrm{DC})$. Let $f: \mathcal{D} \rightarrow \mathcal{D}$ be such that $f(\mathbf{d})<\mathbf{d}$ a.e.; then for some $c, f(\mathbf{d})=\mathbf{c}$ a.e. 
The second verifies a special case of conjecture (b). Call $f: \mathcal{D} \rightarrow \mathcal{D}$ orderpreserving a.e. if and only if there is a $\mathbf{c}$ such that for all $\mathbf{a}, \mathbf{b} \geq \mathbf{c}$, we have that $\mathbf{a} \leq \mathbf{b}$ implies $f(\mathbf{a}) \leq f(\mathbf{b})$.

THEOREM 5. (ZF $+\mathrm{AD}+\mathrm{DC})$. Let $f: \mathcal{D} \rightarrow \mathcal{D}$ be order-preserving a.e. and such that $\mathbf{d}<f(\mathbf{d})$ a.e. Then either

(i) $\exists \alpha<\omega_{1}\left(f(\mathbf{d})=\mathbf{d}^{\alpha}\right.$ a.e. $)$, or

(ii) For a.e. $\mathbf{d}, \forall \alpha<\omega_{1}^{\mathbf{d}}\left(f(\mathbf{d})>\mathbf{d}^{\alpha}\right)$.

(Here $\omega_{1}$ is the least uncountable ordinal, and $\omega_{1}^{\mathbf{d}}$ is the least d-admissible ordinal greater than $\omega$. )

2020 comments. Problem \# 5 is commonly known as Martin's Conjecture. We shall refer to its restriction to uniformly representable functions as the Uniform Martin's Conjecture. Steel had proved part (b) of the Uniform Martin's Conjecture [Ste82A]; for further work in this direction, $c f$. [Bec86]. The partial results by Slaman and Steel listed in the 1983 progress report appear in [SS88] and constitute a proof of the Uniform Martin's Conjecture. Theorem 5 can be improved using results of Woodin [Woo08], so that part (ii) of the conclusion can be strengthened to $f(\mathbf{d})>\mathcal{O}^{\mathbf{d}}$. Kihara and Montalbán recently refined the Uniform Martin's Conjecture to functions from the Turing degrees to the many-one degrees [KM18].

A competing conjecture from the theory of Borel equivalence relations is in conflict with Martin's conjecture: For Polish spaces $X$ and $Y$ and equivalence relations $\equiv$ and $\equiv^{\prime}$ on $X$ and $Y$, respectively, we say that $\equiv$ is Borel reducible to $\equiv^{\prime}$ if and only if there is a Borel function $f: X \rightarrow Y$ such that for all $x, x^{\prime} \in X$ we have

$$
x \equiv x^{\prime} \Longleftrightarrow f(x) \equiv^{\prime} f\left(x^{\prime}\right) .
$$

An equivalence relation on $X$ is Borel if and only if it is a Borel subset of $X \times X$, and it is countable if and only if all its equivalence classes are countable. A countable Borel equivalence relation is universal if and only if all countable Borel equivalence relations are Borel reducible to it. Kechris asked (cf. [Kec92, Problem 17, p. 99]): ${ }^{5}$

Question 6. Is Turing equivalence $\equiv_{\mathrm{T}}$ universal?

Slaman and Steel have also shown that arithmetic equivalence is universal [MSS16, § 2], but the question remains open for Turing equivalence. A positive answer to Kechris's question would contradict Martin's conjecture: if there is a Borel reduction of two disjoint copies of $\equiv_{\mathrm{T}}$ to $\equiv_{\mathrm{T}}$, then the range of one of the copies under the reduction would be a set disjoint from a cone. ${ }^{6}$ A detailed discussion of the current state of knowledge, including a proof of the Slaman-Steel result on arithmetic equivalence, can be found in [MSS16].

\footnotetext{
${ }^{5}$ This question is sometimes stated as a conjecture; $c f$. [DK00, Conjecture, p. 86].

${ }^{6}$ Details can be found in [DK00, second Fact on p. 86].
} 
Montalbán, Reimann, and Slaman, have shown (in unpublished work) that Turing equivalence is not uniformly universal [Sla09].

\section{\# 6. The extent of definable scales.}

\section{Original problem [KMS88A].}

Problem \# 6. Assume $\boldsymbol{\sim}_{1}^{1}-\mathrm{AD}^{\Sigma_{3}}$. Do all $\mathrm{I}^{\Sigma_{2}} \boldsymbol{\sim}_{1}^{1}$ sets admit $\mathrm{HOD}(\mathbb{R})$ scales?

The terminology is explained in Steel's paper [Ste88]. The strongest result in this direction has been Martin's theorem that for $\lambda<\omega_{1}$ a limit ordinal,

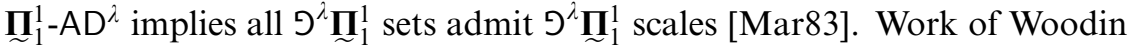
and Steel had shown that a positive answer to \# 6 implies that some form of

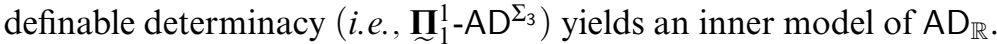

Steel obtained a positive answer to \# 6 in February 1984; his results in this area are described in [Ste88].

2020 comments. There has been a significant amount of additional work on determinacy of long games and regularity of associated sets. In [Ste08C], Steel extends the work published in [Ste88]:

Say that $T$ is an $\omega_{1}$-tree if and only if $T \subseteq \omega^{<\omega_{1}}$ and $T$ is closed under initial segments. ${ }^{7}$ For an $\omega_{1}$-tree $T$, the game $\mathcal{G}(T)$ is the following (closed) game on $\omega$ of length $\omega_{1}$ : For any countable $\alpha$, at stage $\alpha$, player I plays an integer $m_{\alpha}$ and player II replies an integer $n_{\alpha}$. Letting $\langle\cdot, \cdot\rangle$ denote a (natural) pairing function, let $f: \omega_{1} \rightarrow \omega$ be the function defined at any $\alpha$ by $f(\alpha)=\left\langle m_{\alpha}, n_{\alpha}\right\rangle$. Player II wins this run of the game if and only if $f \in[T]$, the set of length- $\omega_{1}$ branches through $T$.

We say that $\omega_{1}$-open-projective determinacy holds if for all $\omega_{1}$-trees $T$ definable over $\mathbf{H}\left(\omega_{1}\right)$ from parameters, the game $\mathcal{G}(T)$ is determined. We let $\supset^{\omega_{1}}$ (open-analytical) be the pointclass of all sets of the form $\supset^{\omega_{1}}(T)$ for such a tree.

THEOREM 7 (Steel). If $\omega_{1}$-open-projective determinacy holds, then the pointclass $\supset^{\omega_{1}}$ (open-analytical) has the scale property.

The determinacy property is called " $\omega_{1}$-open-projective" because an $\omega_{1}$-tree is definable over $\mathbf{H}\left(\omega_{1}\right)$ from parameters if and only if it can be coded by a projective set of reals. In [Nee04], Neeman proved that $\omega_{1}$-open-projective determinacy follows from a traditional large cardinal assumption, viz., that for every real $x$ there is a countable, $\omega_{1}+1$-iterable (coarse) mouse $M$ with $x \in M$ and $M \models \mathrm{ZFC}-\mathrm{P}+$ "there is a measurable Woodin cardinal", where ZFC - P denotes ZFC without the power set axiom. The monograph [Nee04] describes the state of the art in the theory of determinacy of long games around

\footnotetext{
${ }^{7}$ The usual definition implies that $\omega_{1}$-trees have height $\omega_{1}$ and that each level be countable. The present form weakens both requirements but keeps that each node has at most countably many immediate successors, while simultaneously providing a uniform way of ensuring the countability of each of these sets of successors.
} 
2004, although a few results of Woodin in the area remain unpublished and the field has further developed since then.

\section{\# 7. The Kleene ordinal.}

\section{Original problem [KMS88A].}

PROBLEM \# 7. Let $\kappa$ be the least ordinal not the order type of a prewellordering of $\mathbb{R}$ recursive in Kleene's ${ }^{3} E$ and a real. Assume $\mathrm{AD}^{\mathbf{L}(\mathbb{R})}$. Is $\kappa$ the least weakly inaccessible cardinal?

That the answer is positive is an old conjecture of Moschovakis, who had shown that $\kappa$ is a regular limit of Suslin cardinals [Mos70, Mos78]. Steel showed in [Ste81A] that $\kappa$ is the least regular limit of Suslin cardinals. Thus the problem amounted to bounding the growth of the Suslin cardinals below $\kappa$. Building on work of Kunen and Martin, Jackson had done this for the first $\omega$ Suslin cardinals; this work is described in his long paper [Jac88].

In the fall of 1985, Jackson obtained a positive answer to \# 7. His new work extends the theory presented in [Jac88]. Because of its length and complexity, as of now no one but Jackson has been through this new work.

2020 comments. Jackson's result remains unpublished; he comments:

Steel has developed a "fine structure theory" for $\mathbf{L}(\mathbb{R})$ assuming $Z F+A D$. This suffices to answer certain questions about $\mathbf{L}(\mathbb{R})$, for example, it gives a complete description of the scale property in $\mathbf{L}(\mathbb{R})$. Other problems, however, such as whether every regular cardinal is measurable seem to require a more detailed understanding of $\mathbf{L}(\mathbb{R})$.

Our results provide such a detailed analysis for an initial segment of the $\mathbf{L}_{\alpha}(\mathbb{R})$ hierarchy. Exactly how far this enables one to go is not clear, and is the subject of current investigation. However, the author has verified that the theory extends through the Kleene ordinal $\kappa=\mathrm{o}\left({ }^{3} E\right)$, and in fact, considerably beyond. This analysis is quite involved, however, and has not yet been written up. One consequence is the solution to a problem of Moschovakis, who conjectured in ZF + AD + DC that the Kleene ordinal should be the least inaccessible cardinal (this is the seventh Victoria Delfino problem). [Jac89, p. 80]

As already quoted in our comments to Problem \# 1, Jackson reports in 2010 that he had

extended the analysis further, up to the least inaccessible cardinal in $\mathbf{L}(\mathbb{R})$, although this lengthy analysis has never been written up. [Jac10, p. 1755] 
To the best of our knowledge, no alternative approaches (via the HOD analysis or otherwise) have been suggested. Portions of the analysis have appeared in [Jac91, Jac92, Jac10].

\section{\# 8. Regular cardinals in $\mathrm{L}(\mathbb{R})$.}

\section{Original problem [KMS88A].}

Problem \# 8. Assume $\mathrm{AD}+\mathbf{V}=\mathbf{L}(\mathbb{R})$. Are all regular cardinals below $\Theta$ measurable?

Moschovakis and Kechris had shown, in ZFC $+\mathrm{AD}^{\mathbf{L}(\mathbb{R})}$, that if $\kappa$ is regular (in $\mathbf{V}$, where AC holds!) and $\kappa<\Theta^{\mathbf{L}(\mathbb{R})}$, then $\mathbf{L}(\mathbb{R}) \models$ " $\kappa$ is measurable". This led them to conjecture a positive answer to \# 8. Jackson's detailed analysis of cardinals and measures had verified the conjecture for $\kappa$ below the supremum of the first $\omega$ Suslin cardinals (cf. [Jac88]).

The only progress on this problem since its addition to the list is Jackson's new work cited above, which presumably yields a positive answer to \# 8 for $\kappa$ below the Kleene ordinal.

2020 comments. Problem \# 8 was solved by John Steel using core model techniques, specifically through the beginning of what we now call the HOD analysis. The proof is published as [Ste10, Theorem 8.27].

Steel realised, under the assumption of determinacy, the fragment of the model $\mathbf{H O D}^{\mathbf{L}(\mathbb{R})}$ below $\Theta$ as a fine structural mouse, specifically as the direct limit of a system whose objects are certain countable mice and whose commuting maps are appropriate iterations. Analysis of this system allows us to conclude (combinatorial or descriptive set theoretic) properties of its direct limit from (fine structural) properties of the mice, and many different results have been established this way. In particular:

TheOrem 8 (Steel; [Ste95A]). Assume $\mathrm{AD}^{\mathbf{L}(\mathbb{R})}$ and work in $\mathbf{L}(\mathbb{R})$. Then for every $x \in \mathbb{R}$ and $\kappa<\underbrace{2}_{1}$ such that $\kappa$ is regular in $\mathbf{H O D}(x)$, the following implication holds:

$$
\operatorname{cf}(\kappa)>\omega \text { implies } \mathbf{H O D}(x)=" \kappa \text { is measurable". }
$$

These measures on $\kappa$ in $\mathbf{H O D}(x)$ for different $x$ can be amalgamated via the directed system that guides the iterations mentioned above; the result now follows via reflection; $c f$. also [Ste10, Lemma 8.25]

This analysis of $\mathbf{V}_{\Theta} \cap \mathbf{H O D}^{\mathbf{L}(\mathbb{R})}$ has been extended by Woodin to a full analysis of HOD via a longer directed system, while identifying the correct hybrid rather than purely fine structural mice that make up HOD; $c f$. [SW16]. A similar analysis of the HOD of larger models than $\mathbf{L}(\mathbb{R})$ has become a key tool in recent work in determinacy, in particular, in the proofs of partial versions of the mouse set conjecture (cf. the 2020 comments on Problem \# 11). 
To illustrate the reach of the HOD analysis, we mention some further applications (the list is not exhaustive): Recall that, assuming determinacy, $\kappa_{2 n+1}^{1}$ is the cardinal predecessor of the projective ordinal $\boldsymbol{\delta}_{2 n+1}^{1}$. In [Sar13B, Theorem 5.2.2], Sargsyan proves Woodin's theorem that, under $A D+\mathbf{V}=\mathbf{L}(\mathbb{R})$, for all $n \in \omega, \kappa_{2 n+3}^{1}$ is the least cardinal $\delta$ of HOD such that $\mathbf{M}_{2 n}(\mathbf{H O D} \mid \delta) \models " \delta$ is Woodin." This identifies a purely descriptive set theoretic characterisation of cardinals with a fine structural characterisation, and provides us with precise information of how much large cardinal strength the relevant cardinals retain when passing from $\mathbf{V}$ to nice inner models. In [Sar14], Sargsyan uses the HOD analysis to prove the strong partition property of $\boldsymbol{\delta}_{1}^{2}$, a result first established in [KKMW81]. In [Nee07B], Neeman uses the analysis to provide a characterisation of supercompactness measures for $\omega_{1}$ in $\mathbf{L}(\mathbb{R})$. In [JKSW14], the authors use the analysis to prove Woodin's result that, under $A D+\mathbf{V}=\mathbf{L}(\mathbb{R})$, every uncountable cardinal below $\Theta$ is Jónsson and, if its cofinality is $\omega$, then it is even Rowbottom. This drastically extends previous results of Kleinberg [Kle77] and their generalisation by the second author [Löw02].

\section{\# 9. Large cardinals implying determinacy.}

\section{Original problem [KMS88A].}

Problem \#9. Does the existence of a nontrivial, elementary $j: \mathbf{V}_{\lambda+1} \rightarrow \mathbf{V}_{\lambda+1}$ imply $\underset{\sim}{\mathbf{\Pi}} 1$ determinacy?

The world view embodied in the statements of this and the succeeding problem was seriously mistaken. That view was inspired by Martin's result that the existence of a nontrivial, $\Sigma_{1}$-elementary $j: \mathbf{V}_{\lambda+1} \rightarrow \mathbf{V}_{\lambda+1}$ implies $\boldsymbol{\Pi}_{2}^{1}$ determinacy [Mar80], together with work of Mitchell [Mit79] which promised to lead to a proof that nothing much weaker than the existence of such an embedding would imply ${\underset{\sim}{2}}_{2}^{1}$ determinacy. Martin naturally conjectured that a nontrivial, fully elementary $j: \mathbf{V}_{\lambda+1} \rightarrow \mathbf{V}_{\lambda+1}$ would yield PD; hence the inclusion of \# 9 on our list.

Partly because this view was so mistaken, progress in this area since 1984 has been dramatic. From February to April of 1984, Woodin showed that the existence of a nontrivial, elementary $j: \mathbf{L}\left(\mathbf{V}_{\lambda+1}\right) \rightarrow \mathbf{L}\left(\mathbf{V}_{\lambda+1}\right)$ implies PD and in fact $A D^{\mathbf{L}(\mathbb{R})}$. This was still consistent with the view underlying \# 9, and in spirit was a positive answer, although even for ${\underset{\sim}{3}}_{3}^{1}$ determinacy Woodin's result required a hypothesis slightly stronger than allowed in \# 9. However, at about the same time Foreman, Magidor and Shelah [FMS88] developed a powerful new technique for producing generic elementary embeddings under relatively "weak" large cardinal hypotheses such as the existence of supercompact cardinals. Woodin realised at once the potential in their technique and used it to show, in May 1984, that the existence of a supercompact cardinal implies 
all projective sets of reals are Lebesgue measurable. Immediately thereafter, Shelah and Woodin improved this to include all sets in $\mathbf{L}(\mathbb{R})$.

If the relationship between large cardinals and determinacy were to exhibit anything like the pattern it had previously, supercompact cardinals had to imply $A D^{\mathbf{L}(\mathbb{R})}$. In September 1985, Martin and Steel showed that in fact they do (thereby answering \# 9 positively). (Their proof of PD is self-contained. Their proof of $A D^{L(\mathbb{R})}$ requires work done by Woodin using the generic embedding techniques.) The Martin-Steel theorem required much less than supercompactness; e.g., for $\prod_{n+1}^{1}$ determinacy it required the existence of $n$ "Woodin cardinals" with a measurable above them all. [The notion of a "Woodin cardinal" had been isolated by Woodin in his work on generic embeddings; it is a refinement of a notion due to Shelah.] In May-July of 1986, Martin and Steel pushed the theory of inner models for large cardinals far enough to show that the hypothesis of their theorem was best possible: the existence of $n$ Woodin cardinals does not imply ${\underset{\sim}{n+1}}_{n+1}^{1}$ determinacy. More recently, Woodin has obtained relative consistency results in this direction by a different method; cf. Problem \# 10 below.

Unfortunately, with the exception of [FMS88], none of this recent work has been published.

2020 comments. The relationship between determinacy and large cardinals is now well documented. Since this relationship is of fundamental importance to the field and the Cabal, we use this opportunity to give a brief exposition of the developments and the current topics of research.

Woodin cardinals. The mentioned Shelah-Woodin results on Lebesgue measurability of all sets of reals in $\mathbf{L}(\mathbb{R})$ in the presence of large cardinals appear in [SW90]. The paper defines the notions now known as Shelah and Woodin cardinals, although the notation it uses is different, cf. [SW90, p. 384 and Definitions $3.5 \& 4.1$. Since the paper was not published until after the importance of Woodin cardinals had become apparent, the name Woodin cardinal appears in this paper:

We define here two large cardinals: $\operatorname{Pr}_{\mathrm{a}}(\lambda, f), \operatorname{Pr}_{\mathrm{a}}(\lambda)$ by Shelah (Definition 3.5) and $\operatorname{Pr}_{\mathrm{b}}(\lambda)$ by Woodin - now called a Woodin cardinal. [SW90, p. 384]

The mentioned Martin-Steel results appear in [MS88] and [MS89], which also mark the first appearance of the term Woodin cardinal in the literature. The definition of Woodin cardinals given in [SW90] is easily seen to be equivalent to the modern definition: suppose that $\delta$ is an infinite ordinal and that $A \subseteq \mathbf{V}_{\delta}$. A cardinal $\lambda<\delta$ is $<\delta$ - $A$-strong if and only if for any $\mu<\delta$ there is a nontrivial elementary embedding $j: \mathbf{V} \rightarrow M$ with critical point $\lambda$ and such that $j(\lambda)>\mu$, $\mathbf{V}_{\mu} \subset M$, and $j(A) \cap \mathbf{V}_{\mu}=A \cap \mathbf{V}_{\mu}$. The ordinal $\delta$ is a Woodin cardinal if and only if it is an inaccessible cardinal and for all $A \subseteq \mathbf{V}_{\delta}$ there is a $<\delta$ - $A$-strong cardinal. 
Woodinness was instantly recognised as a pivotal large cardinal notion, and its properties were immediately studied in detail. The realisation that Woodin cardinals form a key step in the development of the inner model program confirmed their importance for the field: comparison of mice is central to the theory of fine structural models; comparisons at the level of cardinals that could be reached by the techniques of the early 1980s were linear and this imposed serious limitations on the nature of the corresponding models, e.g., all of them admitted $\Delta_{3}^{1}$ well-orderings of their set of reals. As a consequence, none of them could be models of projective determinacy. Thus, if inner model theory had any hope of reaching supercompact cardinals, essential changes were needed.

The crucial change connected to Woodin cardinals was the increase in the complexity of the comparison process from linear iterations to what are now called iteration trees. The development of the appropriate fine structure followed shortly thereafter [MaS94, MiS94] and led to the precise determination of the effect of Woodin cardinals on the complexity of the reals present in canonical inner models, on the amount of determinacy outright provable or provably consistent, and on the amount of correctness that a model would satisfy or that could be forced of an iterate of the model. As a consequence, the set theoretic landscape transformed significantly thanks to the introduction of Woodin cardinals.

Determinacy from large cardinals. The Martin-Steel theorem mentioned in the original problem, " $n$ Woodin cardinals and a measurable above imply $\operatorname{Det}\left(\boldsymbol{\Pi}_{n+1}^{1}\right)$ ", is published in [MS89]. The optimal result is that if for every real $x$ there is a suitable model $M$ that is iterable and contains $x$ and $n$ Woodin cardinals, then $\operatorname{Det}\left({\underset{\sim}{n+1}}_{n+1}^{1}\right)$ holds [Nee95].

That assuming just $n$ Woodin cardinals does not suffice follows from inner model theory: from the existence of $n$ Woodin cardinals, a fine structural model with $n$ Woodin cardinals can be obtained, in which the reals admit a $\Delta_{n+2}^{1}$ well-ordering [MaS94, Ste95B], and therefore $\operatorname{Det}\left(\Pi_{n+1}^{1}\right)$ fails in the model by [Kan94, Exercise 27.14].

$A D^{L(R)}$ from infinitely many Woodin cardinals and a measurable cardinal above them is due to Woodin, proved using the methods of stationary tower forcing and an appeal to the main theorem, Theorem 5.11, in Martin-Steel [MS89]. A proof using Woodin's genericity iterations and fine structure instead of stationary tower forcing is due to Steel, and the proof reached in this chapter (using a second form of genericity iterations and no fine structure) is due to Neeman. [Nee10, p. 1880]

These arguments can be pushed much further, and the determinacy of stronger pointclasses than $\wp(\mathbb{R}) \cap \mathbf{L}(\mathbb{R})$ is provable by similar methods from 
large cardinals still in the region of Woodin cardinals (in particular, well before reaching the level of rank-to-rank embeddings or even supercompactness).

The consistency strength of the Axiom of Determinacy. Woodin's derived model theorem shows that infinitely many Woodin cardinals without a measurable above suffice to establish the consistency of determinacy in $\mathbf{L}(\mathbb{R}):{ }^{8}$

THEOREM 9. If $\lambda$ is a limit of Woodin cardinals, $G$ is $\operatorname{Col}(\omega,<\lambda)$-generic over $\mathbf{V}$, and $\mathbb{R}^{*}=\bigcup_{\alpha<\lambda} \mathbb{R} \cap \mathbf{V}[G \mid \alpha]$, then $\mathbf{L}\left(\mathbb{R}^{*}\right)$ is a model of determinacy.

In fact, we have that $\mathbb{R}^{*}=\mathbb{R} \cap \mathbf{V}\left(\mathbb{R}^{*}\right)$ and, letting $\Gamma$ denote the collection of all sets of reals $A \subseteq \mathbb{R}^{*}$ in $\mathbf{V}\left(\mathbb{R}^{*}\right)$ such that $\mathbf{L}\left(A, \mathbb{R}^{*}\right) \models \mathrm{AD}^{+}$, then we have that $\mathbf{L}\left(\Gamma, \mathbb{R}^{*}\right)$ is also a model of determinacy.

Here, $\mathrm{AD}^{+}$denotes Woodin's strengthening of the axiom of determinacy; $c f$. the 2020 comments on Problem \# 14 below.

Conversely, if $A D^{\mathbf{L}(\mathbb{R})}$ holds, then in a forcing extension there is a model of choice with $\omega$ Woodin cardinals (cf. [Ste09, KW10, ST10, Zhu10, Zhu15]).

One of the important early results concerning proofs of the existence of large cardinals in inner models from determinacy is the following theorem $(c f$. [KW10, Theorem 5.1]):

THEOREM 10 (Woodin). Assume $\mathrm{AD}+\mathbf{V}=\mathbf{L}(\mathbb{R})$. Then $\Theta$ is a Woodin cardinal in HOD.

Lightface determinacy. Harrington's results on getting sharps from analytic determinacy are lightface: if $x$ is a real and $\operatorname{Det}\left(\Pi_{1}^{1}(x)\right)$ holds, then $x^{\#}$ exists [Har78].

Moving up to $\Pi_{2}^{1}$, we get that if both pointclasses ${\underset{\sim}{1}}_{1}^{1}$ and $\Pi_{2}^{1}$ are determined, then $\mathbf{M}_{1}^{\#}$ exists and is $\omega_{1}$-iterable [SW16, Corollary 4.17]. In 1995, Woodin claimed the following boldface generalisations of this result: ${ }^{9}$

THEOREM 11. If $\operatorname{Det}\left(\mathbf{\Pi}_{n+1}^{1}\right)$ holds, then $\mathbf{M}_{n}^{\#}(x)$ exists and is $\omega_{1}$-iterable for all reals $x$.

The result remained unpublished until [MSW16], where the following strengthening is established:

THEOREM 12 (Woodin). Assume $\operatorname{Det}\left(\Pi_{n+1}^{1}\right)$ and $\operatorname{Det}\left(\boldsymbol{\Pi}_{n}^{1}\right)$. If there is no ${\underset{\sim}{n+2}}_{n+2}^{1}$ sequence of length $\omega_{1}$ of distinct reals, then $\mathbf{M}_{n}^{\#}$ exists and is $\omega_{1}$-iterable.

The proof uses inner model theory and relativizes to give Theorem 11.

In [MSW16, §4.2], the authors further conjecture the strengthening of Theorem 12 where the assumption about the existence of uncountable sequences of reals is removed. For $n=1$, this is [SW16, Corollary 4.17] mentioned above. The conjecture remains open in general, but was settled affirmatively by Zhu for odd numbers in [Zhu16].

\footnotetext{
${ }^{8}$ Appropriate weakenings hold for finitely many Woodin cardinals; e.g., if $\delta$ is Woodin and $G$ is $\operatorname{Col}(\omega, \delta)$-generic over $\mathbf{V}$, then $\operatorname{Det}\left(\Delta_{2}^{1}\right)$ holds in $\mathbf{V}[G]$ [Nee95, Corollary 2.3].

${ }^{9} C f$. [Nee95, p. 328] and [Nee04, p. 9].
} 
The Solovay sequence. Theorem 10 has been significantly generalised and is part of the HOD analysis mentioned in connection with Problem \# 8. In [Sol78B], Solovay introduced the Solovay sequence $\left\langle\Theta_{\alpha} \mid \alpha \leq \Omega\right\rangle$ as a way of measuring the strength of determinacy models: We assume determinacy and let $\Theta_{0}$ is the supremum of all ordinals $\alpha$ for which there is an ordinal definable pre-wellordering of a subset of $\mathbb{R}$ of length $\alpha$. If $\Theta_{\alpha}$ is defined for all $\alpha<\beta$, and $\beta$ is limit, then $\Theta_{\beta}$ is defined as their supremum. Finally, if $\Theta_{\alpha}$ is defined and is less than $\Theta$, then $\Theta_{\alpha+1}$ is the supremum of the lengths of all pre-wellorderings of subsets of $\mathbb{R}$ that are definable from ordinals and a set of reals of Wadge rank $\Theta_{\alpha}$. The sequence ends once an ordinal $\Omega$ is reached such that $\Theta_{\Omega}=\Theta$.

In $\mathbf{L}(\mathbb{R})$, we have that $\Theta=\Theta_{0}$, but longer sequences are possible and correspond to models of stronger versions of determinacy. It turns out that all $\Theta_{\alpha+1}$ are Woodin cardinals in HOD. The situation at limit ordinals is more delicate and still being explored; $c f$. [Sar15]. Conversely, starting with models with many Woodin cardinals, the derived model construction provides us with models of strong versions of determinacy; $c f .$, e.g., [Ste08A].

Very large cardinals. Although no longer relevant to the goal of deriving determinacy from large cardinals, Woodin's original approach led to the development of the theory of large cardinals past the level of rank-to-rank embeddings. The motivation was the realisation that there was a strong analogy between the theory of $\mathbf{L}(\mathbb{R})$ in the presence of determinacy, and the theory of $\mathbf{L}\left(\mathbf{V}_{\lambda+1}\right)$ in the presence of nontrivial embeddings $j: \mathbf{L}\left(\mathbf{V}_{\lambda+1}\right) \rightarrow \mathbf{L}\left(\mathbf{V}_{\lambda+1}\right)$ with $\lambda$ being the supremum of the associated critical sequence. Some results illustrating this can be found in [Kaf04], where versions of the coding lemma are established. For more recent developments, $c f$., e.g., [Dim11, BKW17].

\section{\# 10. Supercompacts in $\operatorname{HOD}^{\mathrm{L}(\mathbb{R})}$.}

\section{Original problem [KMS88A].}

Problem \# 10. Assume $\mathrm{AD}^{\mathbf{L}(\mathbb{R})}$. Does $\mathbf{H O D}^{\mathbf{L}(\mathbb{R})}$ satisfy "there is a $\kappa$ such that $\kappa$ is $2^{\kappa}$-supercompact"?

Becker and Moschovakis [BM81] had shown that $\mathbf{H O D}^{\mathbf{L}(\mathbb{R})} \models$ "there is a $\kappa$ such that $\mathrm{o}(\kappa)=\kappa^{+}$". Martin (unpublished) then showed $\mathbf{H O D}^{\mathbf{L}(\mathbb{R})} \models$ "there is a $\kappa$ such that $\kappa$ is $\mu$-measurable". Steel (unpublished) then showed $\operatorname{HOD}^{\mathbf{L}(\mathbb{R})} \models$ "there is a $\kappa$ such that $\kappa$ is $\lambda$-strong, where $\lambda>\kappa$ is measurable". Inspired by these results, the Cabal conjectured that the model $\mathbf{H O D}^{\mathbf{L}(\mathbb{R})}$ satisfies all large cardinal hypotheses weaker than that which implies $A D^{\mathbf{L}(\mathbb{R})}$ (which is false in $\mathbf{H O D}^{\mathbf{L}(\mathbb{R})}$ ). Problem \# 10 resulted from our mistaken guess as to what these hypotheses are.

The Woodin-Shelah Theorem that the existence of supercompacts implies all sets in $\mathbf{L}(\mathbb{R})$ are Lebesgue measurable settles \# 10 negatively, since, assuming 
$\mathrm{AD}^{\mathbf{L}(\mathbb{R})}, \mathbf{H O D}^{\mathbf{L}(\mathbb{R})} \models$ "there is a wellorder of $\mathbb{R}$ in $\mathbf{L}(\mathbb{R})$ ". However, except for the mistake about the cardinals involved, the answer to \# 10 is positive. Woodin has recently (February 1987) shown that, assuming $A^{\mathbf{L}(\mathbb{R})}, \mathbf{H O D}^{\mathbf{L}(\mathbb{R})} \models$ "there is a $\kappa$ such that $\kappa$ is a Woodin cardinal", and under the same assumption found a natural submodel of $\mathbf{H O D}^{\mathbf{L}(\mathbb{R})}$ satisfying "there are $\omega$ Woodin cardinals". The work of Martin, Steel and Woodin referred to in the discussion of \# 9, together with further work of Woodin reducing its large cardinal hypothesis, shows that $A D^{\mathbf{L}(\mathbb{R})}$ follows from the existence of $\omega$ Woodin cardinals with a measurable above them all, so that Woodin's recent work is in spirit a positive answer to \# 10.

2020 comments. The remarks we gave on Problem \# 9 apply here as well. The paper [KW10] shows how to find Woodin cardinals in HOD. Assuming strong forms of determinacy, the question of precisely which large cardinals can be present in HOD remains open, with modern research in descriptive inner model theory motivated by the expectation that at least a very large initial segment of the large cardinal hierarchy should be realised within the HOD models of strong models of determinacy [Sar13A].

\section{\# 11. The GCH in $\operatorname{HOD}^{\mathrm{L}(\mathbb{R})}$.}

\section{Original problem [KMS88A].}

Problem \# 11. Assume $\mathrm{AD}^{\mathbf{L}(\mathbb{R})}$. Does $\mathbf{H O D}^{\mathrm{L}(\mathbb{R})}$ satisfy the $\mathrm{GCH}$ ?

Becker [Bec80] has shown that, assuming $\mathrm{AD}^{\mathbf{L}(\mathbb{R})}, \mathbf{H O D}^{\mathbf{L}(\mathbb{R})} \models 2^{\kappa}=\kappa^{+}$for many cardinals $\kappa$. There has been little progress on this question since January 1984. Woodin's recent work on large cardinals in $\mathbf{H O D}^{\mathbf{L}(\mathbb{R})}$ does show that

$$
\left.\mathbf{H O D}^{\mathbf{L}(\mathbb{R})} \models \text { “( } \stackrel{(}{1}_{1}^{2}\right)^{\mathbf{L}(\mathbb{R})} \text { is } \Theta^{\mathbf{L}(\mathbb{R})} \text {-strong”. }
$$

It follows by an easy reflection argument that if $\mathbf{H O D}^{\mathbf{L}(\mathbb{R})}$ satisfies the $\mathrm{GCH}$ below $\left(\boldsymbol{\delta}_{1}^{2}\right)^{\mathbf{L}(\mathbb{R})}$, then it satisfies the GCH.

2020 comments. Steel's analysis of HOD below $\boldsymbol{\delta}_{1}^{2}$, mentioned in the solution to Problem \# 8, also solves \# 11, cf. [Ste10, Corollary 8.22]. Beyond the fine structural analysis, Steel's argument uses the result mentioned in the original wording of the problem, that under $A D, \underset{\sim}{\delta_{1}^{2}}$ is strong up to $\Theta$ in $\mathbf{H O D}^{\mathbf{L}(\mathbb{R})} ; c f .[\mathrm{KW} 10]$. It also uses that there is a set $P \subseteq \Theta$ in $\mathbf{L}(\mathbb{R})$ such that $\mathbf{H O D}^{\mathbf{L}(\mathbb{R})}=\mathbf{L}(P)$. Both these results are due to Woodin. The second follows from the analysis of the Vopěnka algebra; $c f$. [SW16].

The argument generalises to the HOD of larger models of determinacy, as long as the models allow a version of the HOD analysis. At the moment, this falls within the region below a Woodin limit of Woodin cardinals or, in terms of determinacy assumptions, somewhere in the neighbourhood of $A D_{\mathbb{R}}+$ " $\Theta$ is 
regular" [Sar15, Tra14, AS19, ST16]. The expectation is that the result should hold in general.

\section{\# 12. Projective uniformisation, measure, and category.}

\section{Original problem [KMS88A].}

PROBLEM \# 12. Does the theory ZFC+ "Every projective relation can be uniformised by a projective function" + "Every projective set is Lebesgue measurable and has the property of Baire" prove PD?

Woodin [Woo82] showed that the theory in question proves $\forall x \subseteq \omega\left(x^{\dagger}\right.$ exists) and more in this direction, together with some other consequences of $\mathrm{PD}$, and conjectured a positive answer to \# 12 .

There has been no direct progress on this problem since 1984 .

2020 comments. Although the expectation was a positive answer, Problem \# 12 was solved negatively by Steel in 1997 . The precise strength of the theory in question is that of ZFC together with the existence of a cardinal $\delta$ with countable cofinality that is the limit of cardinals that are $\delta$-strong.

Details can be found as handwritten notes by Schindler [Sch99], and in Philipp Doebler's Master's thesis [Doe06]. Steel showed that the large cardinal mentioned above suffices to produce a model of the theory under consideration, and Schindler proved that this is indeed an equiconsistency, $c f$. [Sch02, Theorem 9.1].

We sketch Steel's argument. If there is a cardinal $\delta$ as required, then there is a minimal, fully iterable, fine structural inner model $\mathbf{L}[E]$ witnessing that there is such a cardinal $\delta$; this model admits a $\Sigma_{\sim}^{1}$ well-ordering of its reals and this means that $\operatorname{Det}\left(\Delta_{2}^{1}\right)$ must fail.

Steel argues by forcing with $\operatorname{Col}(\omega, \delta)$ over $\mathbf{L}[E]$. In the resulting model, all projective sets are Lebesgue measurable, and have the Baire property and we have projective uniformisation. Furthermore, $\mathbf{L}[E]$ is the core model of any of its forcing extensions, and thus $\mathbf{L}[E] \prec_{\Sigma_{3}^{1}} \mathbf{L}[E][G]$. Since $\operatorname{Det}\left({\underset{\Delta}{2}}_{2}^{1}\right)$ is a

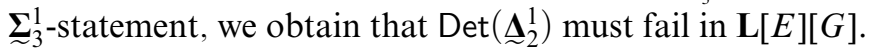

Using the additional assumption that $\mathbb{R}^{\#}$ exists (in order to implement the core model theory of [Ste96]) and results of Schindler on the complexity of $K \cap \mathbf{H}\left(\omega_{1}\right),{ }^{10}$ Hauser and Schindler showed that the theory in Problem \# 12 gives us an inner model with a cardinal $\delta$ and an $\omega$-sequence of cardinals cofinal in $\delta$ and $\delta$-strong [HS00]. Finally, in [Sch02], Schindler shows that, at the level of the theories under consideration, core model theory works without this additional assumption and therefore provides us with a genuine equiconsistency.

${ }^{10} \mathrm{Cf}$. [HS00, Theorems $3.4 \&$ 3.6] which in turn relied on earlier work by Hauser and Hjorth [HH97]. 
From further results in [HS00] and the same argument from [Sch02], we also have that the theory ZF+"Every projective relation can be uniformised by a projective function" + "Every projective set is Lebesgue measurable and has the property of Baire" (i.e., the theory considered in Problem \# 12 without the Axiom of Choice) gives us an inner model with a cardinal $\delta$ of cofinality $\omega$ that is the limit of cardinals that are $\lambda$-strong for all $\lambda<\delta$. This is also an equiconsistency, as can be verified by starting with the corresponding minimal $\mathbf{L}[E]$ model for this large cardinal assumption, and forcing now with the symmetric collapse of $\delta$.

Two variations of Problem \# 12 remain open: ${ }^{11}$

In the first variation, we strengthen the theory by changing the assumption of projective uniformisation with its level-by-level version, namely, that for each $n$, any ${\underset{\sim}{2 n+1}}_{2 n}^{1}$ subset of $\mathbb{R}^{2}$ can be uniformised by a function with a ${\underset{\sim}{2 n+1}}_{2}^{1}$ graph. Steel has shown that this version implies $\operatorname{Det}\left({\underset{\sim}{2}}_{2}^{1}\right) ; c f$. [Ste96, Corollary 7.14].

In the second variation, we replace the assumption with its lightface version, i.e., that all lightface projective subsets of $\mathbb{R}^{2}$ can be uniformised by a function with a lightface projective graph.

\section{\# 13. The cofinal branches hypothesis.}

The cofinal branches hypothesis, introduced by Martin and Steel [MaS94, pp. 50-53], is the statement that every countable iteration tree on $\mathbf{V}$ has at least one cofinal well-founded branch; we write $\mathrm{CBH}$ for this statement.

\section{Problem \# 13. Does CBH hold?}

The unique branches hypothesis, UBH, also introduced by Martin-Steel [MaS94], is the statement that every countable iteration tree on $\mathbf{V}$ has at most one cofinal well-founded branch. As long as the iteration tree $\mathcal{T}$ under consideration is sufficiently closed, UBH for $\mathcal{T}$ implies $\mathrm{CBH}$ for $\mathcal{T}$.

2020 comments. A few years after the problem was formulated, Woodin refuted UBH using large cardinals at the level of embeddings $j: \mathbf{V}_{\lambda} \rightarrow \mathbf{V}_{\lambda}$. Later, in 1999, he also refuted $\mathrm{CBH}$, from the existence of a supercompact with a Woodin above, showing from these assumptions that there is an iteration tree of length $\omega^{2}$ with no cofinal well-founded branch. The tree is formed by an ultrapower by an extender, followed by an $\omega$-sequence of alternating chains on the ultrapower model.

The argument also refutes UBH from the same assumptions, the counterexample being a single ultrapower, now followed by an alternating chain on the ultrapower model, both of whose branches are well-founded.

Details for the case of UBH were presented by Woodin at a meeting at the American Institute of Mathematics (AIM) in December 2004. Later, Neeman

\footnotetext{
${ }^{11} C f$. [Hau00] for more information on both of them.
} 
and Steel significantly lowered the large cardinal assumption needed for both results, to something weaker than the existence of a cardinal strong past a Woodin. More precisely, Neeman and Steel obtained their counterexamples (using the same tree structure as in Woodin's results) from the assumption that there exists a cardinal $\delta$ and an extender $F$ such that $F$ has critical point below $\delta$, support $\delta$, and is $\delta$-strong, and $\delta$ is Woodin in the smallest admissible set containing $\mathbf{V}_{\delta} \cup\{F\}$.

Details, including a discussion of revised versions of both hypotheses that remain open, together with partial positive results, can be found in [NS06].

\section{\# 14. $\infty$-Borel sets.}

Informally, a set is $\infty$-Borel if it can be generated from open sets by closing under the operations of complementation and well-ordered union. Since we are in a choiceless context, we need to give the formal definition in terms of $\infty$-Borel codes. In analogy to standard Borel codes, we define the class of $\infty$-Borel codes by recursion as follows: a tree $T$ is an $\infty$-Borel code if and only if

(i) either $T=\{\langle n\rangle\}$ for some $n \in \omega$,

(ii) or $T=\bigvee_{\alpha} T_{\alpha}:=\{\langle\bigvee, \alpha\rangle\urcorner t: \alpha<\tau$ and $\left.t \in T_{\alpha}\right\}$, where $\tau$ is an ordinal, and each $T_{\alpha} \in \mathrm{BC}$,

(iii) or $\left.T=\neg T^{\prime}:=\{\langle\neg\rangle\urcorner t: t \in T^{\prime}\right\}$, where $T^{\prime} \in \mathrm{BC}$.

Now fix a bijection $\ulcorner.\urcorner:, \omega^{2} \rightarrow \omega$; given $T \in \mathrm{BC}$, we define its interpretation by recursion via

(i) $A_{T}=\left\{x \in{ }^{\omega} \omega: x(k)=\ell\right\}$ if $T=\{\langle\ulcorner k, \ell\urcorner\rangle\}$,

(ii) $A_{T}=\bigcup_{\alpha<\tau} A_{T_{\alpha}}$ if $T=\bigvee_{\alpha} T_{\alpha}$, and

(iii) $A_{T}={ }^{\omega} \omega \backslash A_{T^{\prime}}$ if $T=\neg T^{\prime}$.

Then we say that a set $A$ is $\infty$-Borel if and only if there is an $\infty$-Borel code $T$ such that $A=A_{T}$.

Problem \# 14. Does AD imply that all sets of reals are $\infty$-Borel?

A possibly weaker version of the problem is: Does $A D+D C_{\mathbb{R}}$ imply that all sets of reals are $\infty$-Borel? ${ }^{12}$

2020 comments. Both versions of Problem \# 14 are open. The problem is now considered part of the question whether Woodin's $A D^{+}$is equivalent to $A D$.

In order to define $A D^{+}$, we first need to formulate the concept of ordinal determinacy: if $\lambda<\Theta$, we endow $\lambda$ with the discrete topology, and consider the product topology on ${ }^{\omega} \lambda$. Given a set $A \subseteq{ }^{\omega} \omega$ and a function $f:{ }^{\omega} \lambda \rightarrow{ }^{\omega} \omega$, we consider the game $\mathrm{G}(f, A)$ to be the game of length $\omega$ on $\lambda$ with payoff set

\footnotetext{
${ }^{12}$ The axiom $\mathrm{DC}_{\mathbb{R}}$, or (more precisely) $\mathrm{DC}_{\omega}(\mathbb{R})$, is the statement that whenever $R \subseteq \mathbb{R}^{2}$ satisfies that for any real $x$ there is a $y$ with $x R y$, then there is a function $f: \omega \rightarrow \mathbb{R}$ such that $f(n) R f(n+1)$ for all $n$. Equivalently, any tree $T$ on a subset of $\mathbb{R}$ with no end nodes has an infinite branch.
} 
$f^{-1}[A]$. We say that ordinal determinacy holds if for any $\lambda<\Theta$, any continuous $f:{ }^{\omega} \lambda \rightarrow{ }^{\omega} \omega$, and any set of reals $A$, the game $\mathrm{G}(f, A)$ is determined. Now $\mathrm{AD}^{+}$ is the conjunction of "All sets are $\infty$-Borel", $\mathrm{DC}_{\mathbb{R}}$, and ordinal determinacy. ${ }^{13}$ It is not known whether any of the three components of $A D^{+}$follows from $A D$.

It is known that $A D^{+}$holds in natural models of determinacy, such as models of the form $\mathbf{L}(\wp(\mathbb{R}))$ obtained through the derived model construction. Woodin has shown that $A D_{\mathbb{R}}$ (in fact, $A D+$ Uniformisation) implies that all sets of reals are $\infty$-Borel (cf., e.g., [IW09, Theorem 4.10]).

The problem is closely connected to a number of other famous open problems in the area:

If every set of reals is $\infty$-Borel and there is no uncountable sequence of distinct reals, then all sets of reals are Ramsey, Lebesgue measurable, have the Baire property, and the perfect set property ( $c f$. [CK11]); therefore a positive answer for Problem \# 14 would imply that AD implies that every set of reals is Ramsey (cf. [Kan94, Question 27.18]).

In unpublished work, Woodin has shown that from the consistency of $Z F+D C+A D+$ "not every set of reals is $\infty$-Borel" one can prove the consistency of $Z F+D C+A D+$ "there exists $\kappa>\Theta$ with the strong partition property". This connects the problem with the open problem whether it is consistent to have a strong partition cardinal above $\Theta$.

\section{REFERENCES}

Arthur W. Apter, Stephen C. Jackson, and Benedikt LöWe

[AJL13] Cofinality and measurability of the first three uncountable cardinals, Transactions of the American Mathematical Society, vol. 365 (2013), no. 1, pp. 59-98.

RACHID ATMAI

[Atm19] An analysis of the models $\mathbf{L}\left[T^{2 n}\right.$, The Journal of Symbolic Logic, vol. 84 (2019), pp. 1-26.

RaChid ATMAi AND GRIGOR SARgSYAN

[AS19] HOD up to $\mathrm{AD}_{\mathbb{R}}+\Theta$ is measurable, Annals of Pure and Applied Logic, vol. 170 (2019), no. 1 , pp. 95-108.

Joan Bagaria, Peter Koellner, and W. Hugh Woodin

[BKW17] Large cardinals beyond choice, 2017, preprint.

James E. Baumgartner, Donald A. Martin, and Saharon Shelah

[BMS84] Axiomatic Set Theory. Proceedings of the AMS-IMS-SIAM joint summer research conference held in Boulder, Colo., June 19-25, 1983, Contemporary Mathematics, vol. 31, American Mathematical Society, 1984.

HOWARD S. BECKER

[Bec80] Thin collections of sets of projective ordinals and analogs of $\mathbf{L}$, Annals of Mathematical Logic, vol. 19 (1980), pp. 205-241.

[Bec86] Inner model operators and the continuum hypothesis, Proceedings of the American Mathematical Society, vol. 96 (1986), no. 1, pp. 126-129.

\footnotetext{
${ }^{13} \mathrm{Cf}$. [CK11, § 2] for an introduction to $\mathrm{AD}^{+}$.
} 
Howard S. Becker and Alexander S. Kechris

[BK84] Sets of ordinals constructible from trees and the third Victoria Delfino problem, in Baumgartner et al. [BMS84], pp. 13-29.

Howard S. BECKER AND YiANNIS N. Moschovakis

[BM81] Measurable cardinals in playful models, in Kechris et al. [CABAL ii], pp. 203-214, reprinted in [CABAL III], pp. 115-125.

STEFAN BOLD

[Bo109] Cardinals as Ultrapowers. A Canonical Measure Analysis under the Axiom of Determinacy, Ph.D. thesis, Rheinische Friedrich-Wilhelms-Universität Bonn, 2009.

STEFAN BoLd AND BENEDiKT LÖWE

[BL07] A simple inductive measure analysis for cardinals under the Axiom of Determinacy, Advances in logic. Papers from the North Texas Logic Conference held at the University of North Texas, Denton, TX, October 8-10, 2004 (Su Gao, Steve Jackson, and Yi Zhang, editors), Contemporary Mathematics, vol. 425, American Mathematical Society, Providence, RI, 2007, pp. 23-41.

ANDRÉs EdUARDo CAICEDO AND RichaRd KETCHERSID

[CK11] A trichotomy theorem in natural models of $\mathrm{AD}^{+}$, Set theory and its applications. Papers from the Annual Boise Extravaganzas (BEST) held in Boise, ID, 1995-2010 (Liljana Babinkostova, Andrés E. Caicedo, Stefan Geschke, and Marion Scheepers, editors), Contemporary Mathematics, vol. 533, American Mathematical Society, 2011, pp. 227-258.

Mark Crawshaw

[Cra85] Explicit Formulas for the Jump of Q-Degrees, Ph.D. thesis, California Institute of Technology, 1985.

VinCENZO DimONTE

[Dim11] Totally non-proper ordinals beyond $\mathbf{L}\left(\mathbf{V}_{\lambda+1}\right)$, Archive for Mathematical Logic, vol. 50 (2011), no. 5-6, pp. 565-584.

PHILIPP DOEBLER

[Doe06] The 12th Delfino Problem and universally Baire sets of reals, Master's thesis, Westfälische Wilhelms-Universität Münster, 2006.

Randall Dougherty and Alexander S. Kechris

[DK00] How many Turing degrees are there?, Computability Theory and its Applications. Current Trends and Open Problems. Proceedings of the AMS-IMS-SIAM Joint Summer Research Conference held at the University of Colorado, Boulder, CO, June 13-17, 1999 (Peter A. Cholak, Steffen Lempp, Manuel Lerman, and Richard A. Shore, editors), Contemporary Mathematics, vol. 257, American Mathematical Society, 2000, pp. 83-94.

Matthew Foreman, Menachem Magidor, and Saharon Shelah

[FMS88] Martin's maximum, saturated ideals and nonregular ultrafilters. I, Annals of Mathematics, vol. 127 (1988), no. 1, pp. 1-47.

DAVID GUASPARI

[Gua73] Thin and wellordered analytical sets, Ph.D. thesis, University of Cambridge, 1973.

David Guaspari and Leo Harrington

[GH76] Characterizing $\mathrm{C}_{3}$ (the largest countable $\Pi_{3}^{1}$ set), Proceedings of the American Mathematical Society, vol. 57 (1976), no. 1, pp. 127-129. 
Leo A. Harrington

[Har78] Analytic determinacy and $0^{\#}$, The Journal of Symbolic Logic, vol. 43 (1978), no. 4, pp. 685-693.

KaI HAUSER

[Hau99] Towards a fine structural representation of the Martin-Solovay tree, 1999, preprint.

[Hau00] Reflections on the last Delfino problem, Logic Colloquium '98. Proceedings of the Annual European Summer Meeting of the Association of Symbolic Logic, held in Prague, Czech Republic, August 9-15, 1998 (Samuel R. Buss, Petr Hájek, and Pavel Pudlák, editors), Lecture Notes in Logic, vol. 13, Cambridge University Press, 2000, pp. 206-225.

Kai Hauser and Greg HJorth

[HH97] Strong cardinal in the core model, Annals of Pure and Applied Logic, vol. 83 (1997), no. 2, pp. $165-198$.

Kai HaUSER AND RALF SCHINDLER

[HS00] Projective uniformization revisited, Annals of Pure and Applied Logic, vol. 103 (2000), no. $1-3$, pp. 109-153.

GREGORY HJORTH

[Hjo95] The size of the ordinal $u_{2}$, Journal of the London Mathematical Society, vol. 52 (1995), no. 3, pp. 417-433.

[Hjo96B] Variations of the Martin-Solovay tree, The Journal of Symbolic Logic, vol. 61 (1996), no. 1 , pp. $40-51$.

Daisuke IKegami and W. Hugh Woodin

[IW09] Real determinacy and real Blackwell determinacy, Institut Mittag-Leffler Report No. 32, 2009/2010, Fall, 2009, available online.

\section{STEPHEN JACKSON}

[Jac88] AD and the projective ordinals, in Kechris et al. [CABAL iv], pp. 117-220, reprinted in [CABAL II], pp. 364-483.

[Jac89] AD and the very fine structure of $\mathbf{L}(\mathbb{R})$, Bulletin of the American Mathematical Society, vol. 21 (1989), no. 1, pp. 77-81.

[Jac91] Admissible Suslin cardinals in $\mathbf{L}(\mathbb{R})$, The Journal of Symbolic Logic, vol. 56 (1991), no. 1, pp. 260-275.

[Jac92] Admissibility and Mahloness in $\mathbf{L}(\mathbb{R})$, Set theory of the continuum. Papers from the workshop held in Berkeley, California, October 16-20, 1989 (Haim Judah, Winfried Just, and Hugh Woodin, editors), Mathematical Sciences Research Institute Publications, vol. 26, SpringerVerlag, 1992, pp. 63-74.

[Jac99] $\boldsymbol{A}$ Computation of $\boldsymbol{\delta}_{5}^{1}$, vol. 140, Memoirs of the AMS, no. 670, American Mathematical Society, July 1999.

[Jac10] Structural consequences of AD, in Kanamori and Foreman [KF10], pp. 1753-1876.

Stephen Jackson, Richard Ketchersid, Farmer Schlutzenberg, and W. Hugh Woodin

[JKSW14] Determinacy and Jónsson cardinals in $\mathbf{L}(\mathbb{R})$, The Journal of Symbolic Logic, vol. 79 (2014), no. 4, pp. 1184-1198.

STEPHEN JACKSON AND FARID KHAFIZOV

[JK16] Descriptions and cardinals below $\boldsymbol{\delta}_{5}^{1}$, The Journal of Symbolic Logic, vol. 81 (2016), no. 4, pp. 1177-1224.

Steve JaCKson AND Benedikt LöWE

[JL13] Canonical measure assignments, The Journal of Symbolic Logic, vol. 78 (2013), no. 2, pp. 403-424. 
Ronald B. JENSEN AND John R. STEEL

[JS13] K without the measurable, The Journal of Symbolic Logic, vol. 78 (2013), no. 3, pp. 708-734.

George KafKoulis

[Kaf04] Coding lemmata in $\mathbf{L}\left(\mathbf{V}_{\lambda+1}\right)$, Archive for Mathematical Logic, vol. 43 (2004), no. 2, pp. 193-213.

AKIHIRO KANAMORI

[Kan94] The Higher Infinite. Large Cardinals in Set Theory from Their Beginnings, Perspectives in Mathematical Logic, Springer-Verlag, Berlin, 1994.

AKIHIRo KanAmori AND Matthew Foreman

[KF10] Handbook of Set Theory, Springer-Verlag, 2010.

AleXANDER S. Kechris

[Kec75] The theory of countable analytical sets, Transactions of the American Mathematical Society, vol. 202 (1975), pp. 259-297.

[Kec81] Homogeneous trees and projective scales, in Kechris et al. [CABAL ii], pp. 33-74, reprinted in [CABAL II], pp. 270-303.

[Kec92] The structure of Borel equivalence relations in polish spaces, Set Theory of the Continuum. Papers from the workshop held in Berkeley, California, October 16-20, 1989 (Haim Judah, Winfried Just, and Hugh Woodin, editors), Mathematical Sciences Research Institute Publications, vol. 26, Springer-Verlag, 1992, pp. 89-102.

Alexander S. Kechris, Eugene M. Kleinderg, Yiannis N. Moschovakis, and W. H. Woodin [KKMW81] The axiom of determinacy, strong partition properties, and nonsingular measures, in Kechris et al. [CABAL ii], pp. 75-99, reprinted in [CABAL I], pp. 333-354.

Alexander S. Kechris, Benedikt Löwe, and John R. Steel

[CABAL I] Games, Scales, and Suslin cardinals: the Cabal Seminar, volume I, Lecture Notes in Logic, vol. 31, Cambridge University Press, 2008.

[CABAL II] Wadge Degrees and Projective Ordinals: the Cabal Seminar, volume II, Lecture Notes in Logic, vol. 37, Cambridge University Press, 2012.

[CABAL III] Ordinal Definability and Recursion Theory: the Cabal Seminar, volume III, Lecture Notes in Logic, vol. 43, Cambridge University Press, 2016.

Alexander S. Kechris and Donald A. Martin

[KM78] On the theory of $\Pi_{3}^{1}$ sets of reals, Bulletin of the American Mathematical Society, vol. 84 (1978), no. 1, pp. 149-151.

Alexander S. Kechris, Donald A. Martin, and Yiannis N. Moschovakis

[KMM81A] Appendix: Progress report on the Victoria Delfino problems, in Cabal Seminar 77-79 [CABAL ii], pp. 273-274.

[CABAL ii] Cabal Seminar 77-79, Lecture Notes in Mathematics, vol. 839, Berlin, Springer-Verlag, 1981.

[KMM83A] Appendix: Progress report on the Victoria Delfino problems, in Cabal Seminar 79-81 [CABAL iii], pp. 283-284.

[CABAL iii] Cabal Seminar 79-81, Lecture Notes in Mathematics, vol. 1019, Berlin, Springer-Verlag, 1983.

Alexander S. Kechris, Donald A. Martin, and John R. Steel

[KMS88A] Appendix: Victoria Delfino problems II, in Cabal Seminar 81-85 [CABAL iv], pp. 221224.

[CABAL iv] Cabal Seminar 81-85, Lecture Notes in Mathematics, vol. 1333, Berlin, Springer-Verlag, 1988. 
AleXander S. Kechris and Yiannis N. Moschovakis

[KM78A] Appendix. The Victoria Delfino problems, in Cabal Seminar 76-77 [CABAL i], pp. 279-282.

[CABAL i] Cabal Seminar 76-77, Lecture Notes in Mathematics, vol. 689, Berlin, Springer-Verlag, 1978.

TAKaYuki KiHARa and ANTONio Montalbán

[KM18] The uniform Martin's conjecture for many-one degrees, Transactions of the American Mathematical Society, vol. 370 (2018), no. 12, pp. 9025-9044.

EUGENE M. KLEINBERG

[Kle77] Infinitary Combinatorics and the Axiom of Determinateness, Lecture Notes in Mathematics, vol. 612, Springer-Verlag, 1977.

Peter Koellner and W. Hugh Woodin

[KW10] Large cardinals from determinacy, in Kanamori and Foreman [KF10], pp. 1951-2119.

BENEDIKT LÖWE

[Löw02] Kleinberg sequences and partition cardinals below $\boldsymbol{\delta}_{5}^{1}$, Fundamenta Mathematicae, vol. 171 (2002), no. 1, pp. 69-76.

Andrew Marks, Theodore A. Slaman, and John R. Steel

[MSS16] Martin's conjecture, arithmetic equivalence, and countable Borel equivalence relations, in Kechris et al. [CABAL III], pp. 493-520.

Donald A. Martin

[Mar80] Infinite games, Proceedings of the International Congress of Mathematicatians, Helsinki 1978 (Olli Lehto, editor), Academia Scientiarum Fennica, 1980, pp. 269-273.

[Mar83] The real game quantifier propagates scales, in Kechris et al. [CABAL iii], pp. 157-171, reprinted in [CABAL I], pp. 209-222.

[Mar] AD and the normal measures on $\boldsymbol{\delta}_{3}^{1}$, unpublished, undated.

Donald A. Martin and John R. STEel

[MS83] The extent of scales in $\mathbf{L}(\mathbb{R})$, in Kechris et al. [CABAL iii], pp. 86-96, reprinted in [CABAL I], pp. $110-120$.

[MS88] Projective determinacy, Proceedings of the National Academy of Sciences of the United States of America, vol. 85 (1988), no. 18, pp. 6582-6586.

[MS89] A proof of projective determinacy, Journal of the American Mathematical Society, vol. 2 (1989), no. 1, pp. 71-125.

[MaS94] Iteration trees, Journal of the American Mathematical Society, vol. 7 (1994), no. 1, pp. $1-73$.

WiLliam J. Mitchell

[Mit79] Hypermeasurable cardinals, Logic Colloquium '78. Proceedings of the Colloquium held in Mons, August 24-September 1, 1978 (Maurice Boffa, Dirk van Dalen, and Kenneth McAloon, editors), Studies in Logic and the Foundations of Mathematics, vol. 97, North-Holland, Amsterdam, 1979, pp. 303-316.

William J. Mitchell and John R. STEel

[MiS94] Fine Structure and Iteration Trees, Lecture Notes in Logic, vol. 3, Springer-Verlag, Berlin, 1994.

YIANNIS N. MoschovaKIS

[Mos70] Determinacy and prewellorderings of the continuum, Mathematical Logic and Foundations of Set Theory. Proceedings of an international colloquium held under the auspices of the Israel Academy of Sciences and Humanities, Jerusalem, 11-14 November 1968 (Y. Bar-Hillel, editor), Studies in Logic and the Foundations of Mathematics, North-Holland, Amsterdam-London, 1970, pp. 24-62. 
[Mos78] Inductive scales on inductive sets, in Kechris and Moschovakis [CABAL i], pp. 185-192, reprinted in [CABAL I], pp. 94-101.

[Mos80] Descriptive Set Theory, Studies in Logic and the Foundations of Mathematics, vol. 100, North-Holland, Amsterdam, 1980.

[Mos83] Scales on coinductive sets, in Kechris et al. [CABAL iii], pp. 77-85, reprinted in [CABAL I], pp. 102-109.

SANDra Müller, Ralf Schindler, ANd W. Hugh Woodin

[MSW16] Mice with finitely many Woodin cardinals from optimal determinacy hypotheses, submitted, 2016.

\section{ITAY NeEMAN}

[Nee95] Optimal proofs of determinacy, The Bulletin of Symbolic Logic, vol. 1 (1995), no. 3, pp. 327-339.

[Nee04] The Determinacy of Long Games, de Gruyter Series in Logic and its Applications, vol. 7, Walter de Gruyter, Berlin, 2004.

[Nee07B] Inner models and ultrafilters in $\mathbf{L}(\mathbb{R})$, The Bulletin of Symbolic Logic, vol. 13 (2007), no. 1, pp. 31-53.

[Nee10] Determinacy in $\mathbf{L}(\mathbb{R})$, in Kanamori and Foreman [KF10], pp. 1877-1950.

Itay Neeman and John R. Steel

[NS06] Counterexamples to the unique and cofinal branches hypotheses, The Journal of Symbolic Logic, vol. 71 (2006), no. 3, pp. 977-988.

GERALD E. SACKS

[Sac76] Countable admissible ordinals and hyperdegrees, Advances in Mathematics, vol. 20 (1976), no. 2, pp. 213-262.

Grigor SARGSYAN

[Sar13A] Descriptive inner model theory, The Bulletin of Symbolic Logic, vol. 19 (2013), no. 1, pp. 1-55.

[Sar13B] On the prewellorderings associated with the directed systems of mice, The Journal of Symbolic Logic, vol. 78 (2013), no. 3, pp. 735-763.

[Sar14] An inner model proof of the strong partition property for $\underset{\sim}{\boldsymbol{\delta}_{1}^{2}}$, Notre Dame Journal of Formal Logic, vol. 55 (2014), no. 4, pp. 563-568.

[Sar15] Hod Mice and the Mouse Set Conjecture, vol. 236, Memoirs of the American Mathematical Society, no. 1111, American Mathematical Society, 2015.

Grigor SARgSYAN AND NAm TRANG

[ST16] The Largest Suslin Axiom, 2016, book manuscript, submitted.

RALF SCHINDLER

[Sch99] The Delfino problem \# 12, Talks in Bonn, 4/21/99-4/23/99, handwritten manuscript, 50 pages, available online, 1999.

[Sch02] The core model for almost linear iterations, Annals of Pure and Applied Logic, vol. 116 (2002), no. 1-3, pp. 205-272.

SaHaron SHelah and W. Hugh Woodin

[SW90] Large cardinals imply that every reasonably definable set of reals is Lebesgue measurable, Israel Journal of Mathematics, vol. 70 (1990), no. 3, pp. 381-394.

THEOdore A. SLaman

[Sla09] Degree invariant functions, slides of a talk at the 2009 VIG, available online, 2009.

Theodore A. Slaman and John R. Steel

[SS88] Definable functions on degrees, in Kechris et al. [CABAL iv], pp. 37-55, reprinted in [CABAL III], pp. 458-475. 


\section{ROBERT M. SOLOVAY}

[Sol78B] The independence of DC from AD, in Kechris and Moschovakis [CABAL i], pp. 171-184, reprinted in this volume.

JOHN R. STEEL

[Ste81A] Closure properties of pointclasses, in Kechris et al. [CABAL ii], pp. 147-163, reprinted in [CABAL II], pp. 102-117.

[Ste82A] A classification of jump operators, The Journal of Symbolic Logic, vol. 47 (1982), no. 2, pp. 347-358.

[Ste83A] Scales in $\mathbf{L}(\mathbb{R})$, in Kechris et al. [CABAL iii], pp. 107-156, reprinted in [CABAL I], pp. $130-175$.

[Ste83B] Scales on $\Sigma_{1}^{1}$ sets, in Kechris et al. [CABAL iii], pp. 72-76, reprinted in [CABAL I], pp. 90-93.

[Ste88] Long games, in Kechris et al. [CABAL iv], pp. 56-97, reprinted in [CABAL I], pp. 223-259.

$[\mathrm{Ste} 95 \mathrm{~A}] \mathbf{H O D}^{\mathbf{L}(\mathbb{R})}$ is a core model below $\Theta$, The Bulletin of Symbolic Logic, vol. 1 (1995), no. 1, pp. $75-84$.

[Ste95B] Projectively wellordered inner models, Annals of Pure and Applied Logic, vol. 74 (1995), no. 1, pp. 77-104.

[Ste96] The Core Model Iterability Problem, Lecture Notes in Logic, no. 8, Springer-Verlag, Berlin, 1996.

[Ste08A] Derived models associated to mice, Computational Prospects of Infinity. Part II. Presented Talks (Chitat Chong, Qi Feng, Theodore A. Slaman, W. Hugh Woodin, and Yue Yang, editors), World Scientific, 2008, pp. 105-193.

[Ste08B] Games and scales. Introduction to Part I, in Kechris et al. [CABAL I], pp. 3-27.

[Ste08C] The length- $\omega_{1}$ open game quantifier propagates scales, in Kechris et al. [CABAL I], pp. 260269.

[Ste08D] Scales in $\mathbf{K}(\mathbb{R})$ at the end of a weak gap, The Journal of Symbolic Logic, vol. 73 (2008), no. 2, pp. 369-390.

[Ste08E] Scales in $\mathbf{K}(\mathbb{R})$, in Kechris et al. [CABAL I], pp. 176-208.

[Ste09] The derived model theorem, Logic Colloquium '06. Proc. of the Annual European Conference on Logic of the Association for Symbolic Logic held at the Radboud University, Nijmegen, July 27-August 2, 2006 (S. Barry Cooper, Herman Geuvers, Anand Pillay, and Jouko Väänänen, editors), Lecture Notes in Logic, vol. 19, Association for Symbolic Logic, 2009, pp. 280-327.

[Ste10] An outline of inner model theory, in Kanamori and Foreman [KF10], pp. 1595-1684.

\section{John R. Steel and Nam Trang}

[ST10] $\mathrm{AD}^{+}$, derived models, and $\Sigma_{1}$ reflection, Notes from the first Münster conference on the core model induction and hod mice, available online, 2010.

JoHn R. STEel AND W. Hugh Woodin

[SW16] HOD as a core model, in Kechris et al. [CABAL III], pp. 257-345.

\section{NAm Trang}

[Tra14] HOD in natural models of $\mathrm{AD}^{+}$, Annals of Pure and Applied Logic, vol. 165 (2014), no. 10, pp. $1533-1556$.

W. Hugh Woodin

[Woo82] On the consistency strength of projective uniformization, Proceedings of the Herbrand Symposium. Logic Colloquium '81. Held in Marseille, July 16-24, 1981 (Jacques Stern, editor), Studies in Logic and the Foundations of Mathematics, vol. 107, North-Holland, Amsterdam, 1982, pp. 365-384.

[Woo99] The Axiom of Determinacy, Forcing Axioms, and the Nonstationary Ideal, de Gruyter Series in Logic and its Applications, vol. 1, Walter de Gruyter, Berlin, 1999. 
[Woo08] A tt version of the Posner-Robinson theorem, Computational Prospects of Infinity. Part II. Presented Talks (Chitat Chong, Qi Feng, Theodore A. Slaman, W. Hugh Woodin, and Yue Yang, editors), World Scientific, 2008, pp. 355-392.

YIZHENG ZHU

[Zhu10] The derived model theorem II, Notes on lectures given by $\mathrm{H}$. Woodin at the first Münster conference on the core model induction and hod mice, available online, 2010.

[Zhu15] Realizing an $\mathrm{AD}^{+}$model as a derived model of a premouse, Annals of Pure and Applied Logic, vol. 166 (2015), no. 12, pp. 1275-1364.

[Zhu16] Lightface mice with finitely many Woodin cardinals from optimal determinacy hypotheses, 2016, preprint, arXiv 1610.02352v1.

[Zhu17] The higher sharp I: on $\mathbf{M}_{1}^{\#}, 2017$, preprint, arXiv:1604.00481v4.

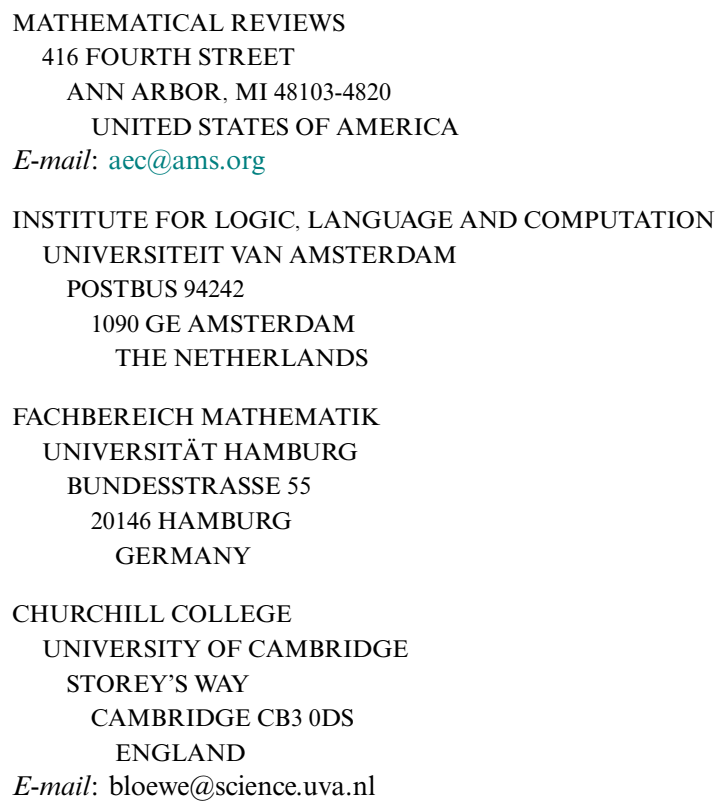

E-mail: bloewe@science.uva.nl 
